Sydro. 5465 $55 / 3$

may 5-7,1914 
Oregon Crat.

$\not 3$

J4m 5 - Mtay 7, 1914.

Btydro. 5\% $65-5513$ 
$2^{8}$

Hydro So. $5-46 \$$ Date, (Revers s , 1914 Sag. Meh. 272 Reel Leads as active. Turns Corn Depth fms.

Sag. Cup Oreminy on Aaspample

Character of
Bottom

Thermos. $\left\{\begin{array}{l}\text { Make } \\ \text { No. }\end{array}\right.$

$$
\text { Corn }\left\{\begin{array}{l}
\text { Instr. } \\
\text { Stem. }
\end{array}\right.
$$

Bottom Reading Cor'd Temp.

$$
\text { Temp. Surf. }\left\{\mathrm{s}^{\circ}\right.
$$

Time Time

$\operatorname{Air}\{$

Density: Surf. fms.

Wind: Direction

Force $3-4$ Baronet. 30,14 Amt of o. State of

weather of

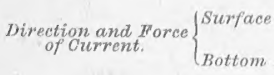

Tide

General Locality Off origan ea ac b

Positionfaquina Right hear $N 8^{\circ} E$ ( thu) distant Chart used: No. Go to Eden

Drift: Direction. Distance

Depth of Hart

Apparatus and Rig used

Time towing $h$ $m$.

Remarks:

Deco offer Been Click

Recorder

Copledong 


\begin{tabular}{|c|c|c|}
\hline SOUNDING WIRE. & $\mathrm{FMC}$ & DREDGING CABLE. \\
\hline P. M. Down. UP. P. M. & TMD & M. Down. UP. \\
\hline
\end{tabular}

usido stsotoo

\begin{tabular}{ll|l|l|l|}
\hline 0 & & \\
\hline
\end{tabular}

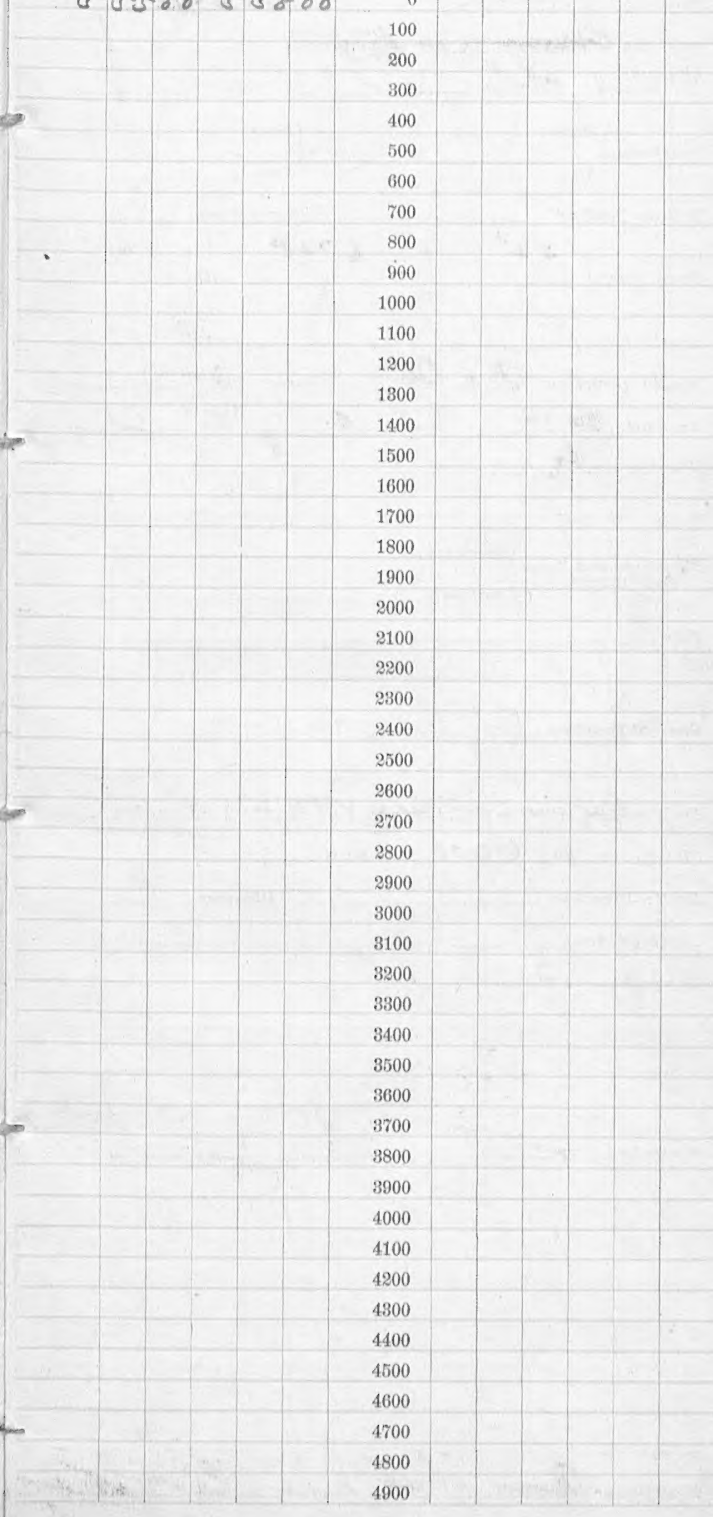


Turns 1.64 exwetcorn

sas. cup Qumaing ar dint, Bot'm Depth d f fms.

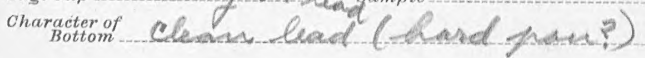

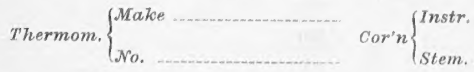

\section{Bottom Reading}

Temp. Surf. $\{-52$

\section{Cor'd Temp.}

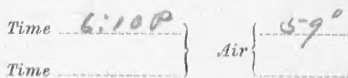

fins.

Density: Surf. Wind: Direction

$2 \times 24$ Force 3

Baromet. 3.4 .14 Amt of weather...

Direction and Force
of Current. $\left\{\begin{array}{l}\text { Surface } \\ \text { Bottom }\end{array}\right.$

Tide

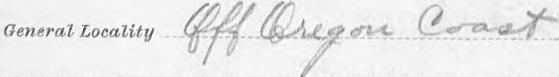

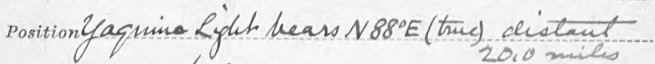
chart used: No. OOOD .... Ed'n

Drift: Direction Distance

Depth of Haut

Apparatus and Rig used

Remarles:

Deck offeer B Bon Recorder 
FMS.

M. Down. UP. $\perp$. 60700.61000

0
DREDGING CABLE.

M. Down. UP, … M.

100

200

300

400

500

600

700

800

900

1000

1100

1200

1300

1400

1500

1600

1700

1800

1900

2000

2100

2200

2300

2400

2500

2600

2700

2800

2900

3000

8100

8200

3300

3400

3500

3600

3700

3800

3900

4000

4100

4200

4300

4400

4500

4600

4700

4800

4900 
32

Hydrogen 1 . No. $5^{-1 / 6} 6$ Date.

Sig. Meh.

Reel.

Allay of ${ }_{19} 14$

Turns 168 Bute te Corn

Shot $2 \mathrm{~S}^{-}$tbs.

Depth 91 fins.

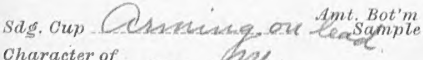

Character of
Bottom

Thermom. $\left\{\begin{array}{l}\text { Make .......... Corn }\left\{\begin{array}{l}\text { Instr. } \\ \text { No. }\end{array} \text { Stem. }\right.\end{array}\right.$

Bottom Reading Cor'd Temp.

Temp. Surf. $\begin{cases}v^{\circ} & \text { Time Gite } \\ & \text { Time }\end{cases}$

Density: Surf.

fms.

Wind: Direction

Force 3

Direction and Force
of Current. $\left\{\begin{array}{l}\text { Surface } \\ \text { Bottom. }\end{array}\right.$

Tide

General Locality Of Oregon Coat Position'yaquina Light hears $\$ 89^{\circ} E$ (tm) didinut Chart used: No. Goo.... Ed'n

Drift: Direction

Distance.

Depth of Haul

Apparatus and Rig used

Time towing 7 . $m$.

Remarks :

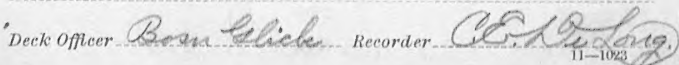


SOUNDING WIRE:

Q M. Down. UP. @. M.

$62300=62700$
FMS DREDGTNG CABLE.

M. Down. UP. M.

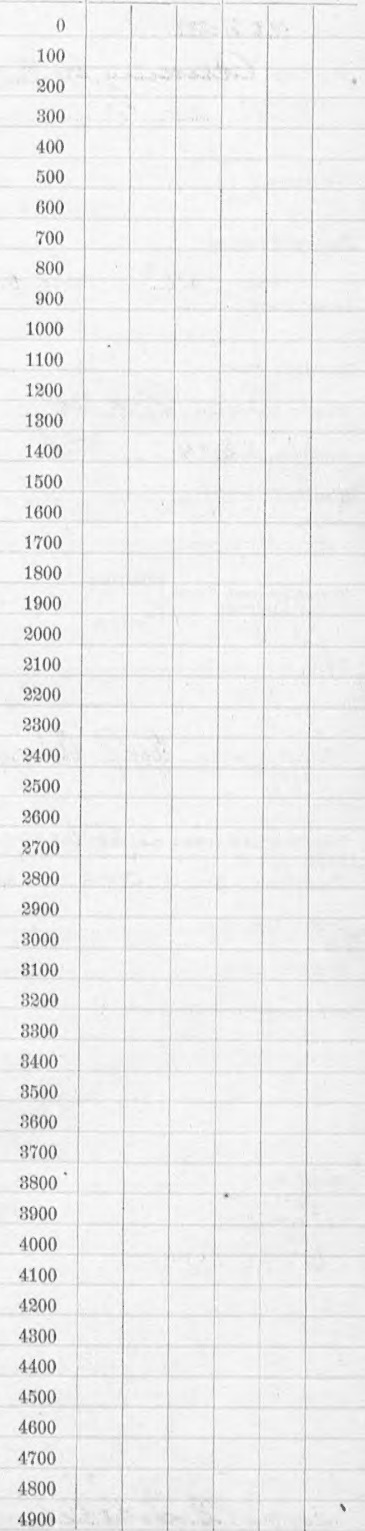


$n_{1} 1$

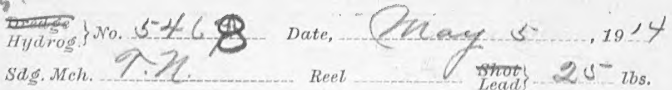
Turns $16 \mathrm{~s}$ metic Reel

sag. Cup Depth go. fms. Character of
Bottom

Thermom. $\left\{\begin{array}{l}\text { Make } \\ \text { No. }\end{array}\right.$

$$
\text { Corn }\left\{\begin{array}{l}
\text { Instr. } \\
\text { Stem. }
\end{array}\right.
$$

Bottom Reading Cor'd Temp.

Temp. Surf. $\left\{-b \nu^{0}\right.$ Time $\left.6: 45^{-}\right\}$Air $\left.\right|_{\text {Time }} ^{4} 66^{\circ}$

Density: Surf. fins.

Wind: Direction

$\times 20$

Force

Baromet. $30 .<4$ Amt. of 35.7

Weather Ley

Direction and Force
of Current.

Tide

General Locality Goff Griqua comet PositionYagmina Light hears S87 E (true) distant Charthed: No. Gods in

Drift: Direction Distance

Depth of Haul

Apparatus and Rig used.

Time towing

h.

$m$.

Remarks

Tine

Wot luck Dy wee

6:45 P.M.

s.2.s

5s:0

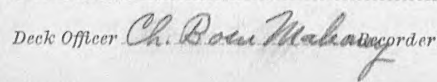


$11-102 ?$

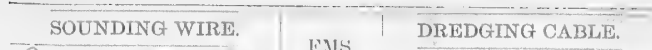

P. M. Down. II. I M.

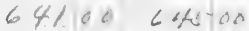

M, Down. U1. M.

67100 CA

100

200

:3011

400

i(1)

(i)10

ino

(i⿱宀⿻三丨日

()(1)

10)( 0

1100

$1 \geqslant 00$

1300

$1.100)$

1:(i)

1(600)

1700

1800

$190(1)$

$\because 000$

2100

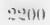

:300

$\because 100$

Pisto

$\because(a)()$

37110

3000

?9:90

:3000

:10k)

:1:00)

:3:i()0)

iifou

i3:500

:iboo

:3700

$: 3800$

:300

10000

.1100

1300

1:300

1100

.1500

f(it)

.1700

.1800

.1900 
Turns/63. ternsts

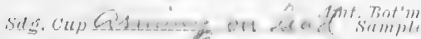

Character of
Bottom

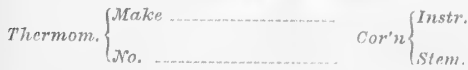

Bottom Readins Cor'd Temp.

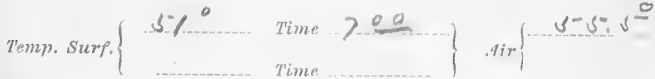

1)ensity: Surf.

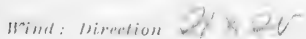

Baromet. 30,14 Amt.of

Weather if 3 Direction and Force
of Current. Bottom

Till

General Tocalily

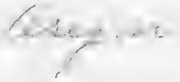

fins.

$$
\text { fiorer } 2-3
$$

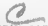

\section{Deptr of ims.}

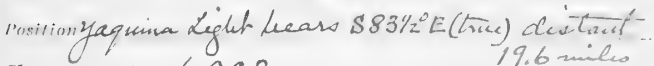

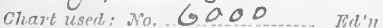

Hrifl: Disereliun

Dislatir

Depth of Haul

Anparatics and his used 
' SOUNDING WIRE.

PaI. Down. H1. PII.

$$
65800>10200
$$

Tils.

DRFDGING CABLE.

$$
\therefore \text { M. Down. Up. M. }
$$

1)( )

)(1)

:io)

fix

500

(ii)

ion)

Sil)

1000

1000

1100

1200

1800

1400

1500

1600

1700

1800

1900

2000

2100

2200

2300

2400

2500

2600

2700

2800

2000

3000

3100

$3: 200$

3300

$: 3400$

3500

3600

$: 3700$

3800

$\$ 3900$

1000)

4100

4:200

4300

4400

4500

4600

4700

4800

4000 


\section{2}

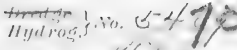

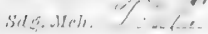

l) II,

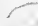

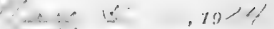

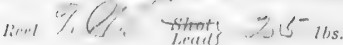

Turns _6. $6 \ldots$ Cor'n. Deptr .... Q Q ..... fims.



Charcicter of $9,4, \ldots+1$.

Thermom. $\left\{\begin{array}{l}\text { Make .............. } \\ \text { No. }\end{array} \int_{\text {Stem. }}^{\text {Instr. }}\right.$

Bottom Reading Cor'd Temp.

Temp. Surf. $\left\{\begin{array}{ll}s-10 & \text { Time } 2,20 \\ \text { Tim. }\end{array}\right\}$ Air $\}$

lonsil!l: surl.

fins.

Hind: Direction XVX IT

Torce $-2=-3$

Baromet. $30<4$ Amt. of

State of

Weather.

Direction and Force
of Current. $\left\{\begin{array}{l}\text { Surfars } \\ \text { Toltom, }\end{array}\right.$

Tiilin

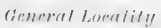
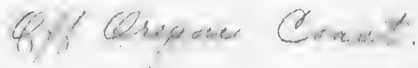

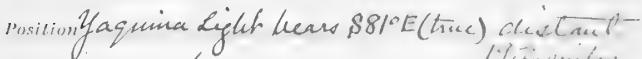
chart used: No,...6000... Mrl't.

11rill: Jirertion Distumen

Depth of Hazl

Apparatus and Rigused

Time towins $m$.

lirmurlis: 
$-11-111: 2 ?$

SOUNDIN(; WTRF.

1.M.

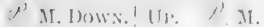

-... M. I) $11 \times$. U. $>1600>2000$

100

20()

:30)

(1)

i(1)

(i)i)

in)

S(I)

!)(H)

1000

110)

Ii)(1)

13(1)

1400

1500

$1600)$

1700

1500

1900

$\because(1)()$

$\because 1140$

is 2()()

13:"(i)(0)

?114)

$\because 5(x)$

1)(i⿰亻)

(1)

$\because 4(11)$

$\because 1)(10)$

$: 1) 0()$

$\because 10)$

:3:0)(1)

ivitur

:) 100

:iint)(

:B(i)o

:in()

:Sotut)

:is)(o)

.1(10)0

11001

1.) $(0)(1)$

.1:30)

$+1100$

tin()

1000

1700

4800

1000 


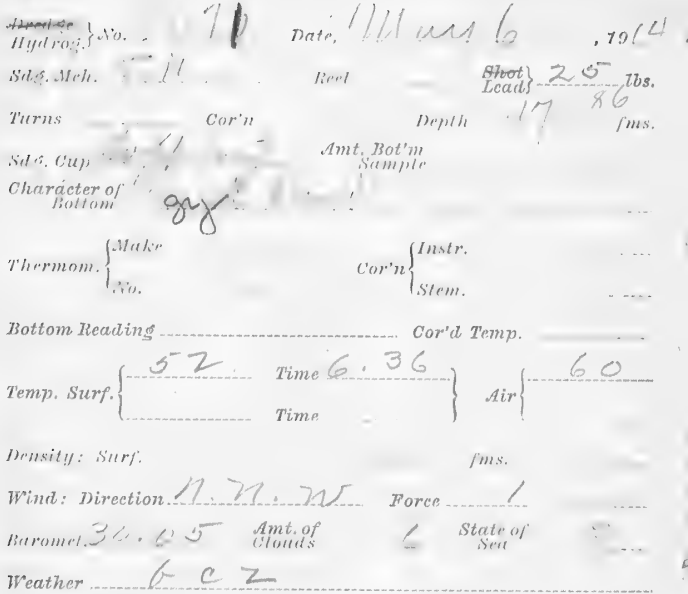

Weather $f c z$

Direction and Forec
of Current. $\left\{\begin{array}{l}\text { Surfuer } \\ \text { liotlom }\end{array}\right.$

Tille ... ..

General hoselill! . :

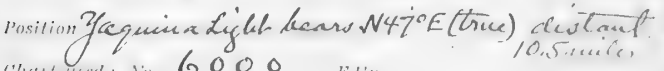
(ithil thited: in. 6000 lid'n

I)rift: Direction

Distun're

Depth of Hazt

Apparatus and Rięused...

Time forming:

h. $m$.

liemerrk:

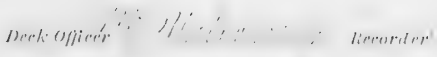


$11-1023$

SOUNDING WIRE

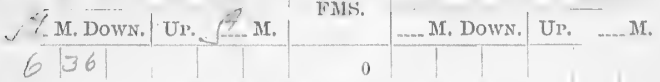

100

200

300

100

500

(;)()

70)

800

900

1000

1100

1200

1300

1400

1500

1600

1700

1800

1900

2000

2100

200

2300

$\because 100$

2500

$\simeq 600$

2700

2800

घ9)(

3000

3100

3200

3300

3400

3500

3000

:เก

(3)

:39011

.4000

(11001

4200

4300

4100

4500

4600

4700

4800

4900 


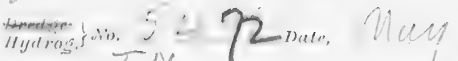
Sag.Meh..T/Y Reel

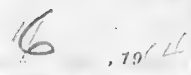

Turns

Cor'n

$12, p, 1 / \mathrm{t}$

Yyhntt
lenuls

का

Sit?. Ciuj,

(liaraclero) liottom

Thermom. $\left\{\begin{array}{l}\text { Wukr } \\ \text { No. }\end{array}\right.$

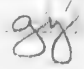
Aml. Bot'm
Sammit

Bottom Reading

$$
\text { Cor'n }\left\{\begin{array}{l}
\text { Instr. } \\
\text { Sterm. }
\end{array}\right.
$$

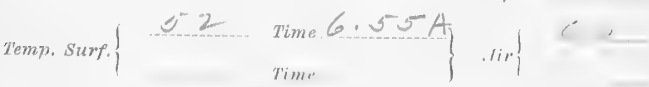

Density: Surf. fims.

Wind: Direction - $1,1 / 22$ Foree

Baromet. 30,05 Amt. of Gtate of S S

Weather C. $c z$ Direction and Force
of Current. $\left\{\begin{array}{l}\text { Surface. } \\ \text { Bottom }\end{array}\right.$

Tirlr.

Generel Lerality

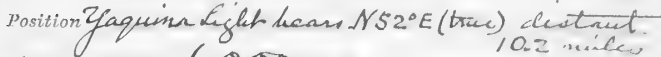

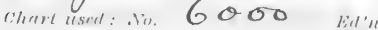

Hrifl: Jimelinn l)istrHer

Depth of Huut

Apparatus and Rig used

liemerlis: 
SOUNDINA WIIRL.

D)REDAIN( (ABLE

M. DOWN. U1: FNS.

M. Dow. U1. .II. $6 \sqrt{5}$

0

100

200

:3(1)

400

500

(i)

700

800

900

1000

1100

1200

1800

1400

1500

1600

1700

1800

1900

2000

2100

2200

2300

2400

2500

2600

2700

2800

2900

3000

3100

3200

3300

8400

3500

3600

3700

3800

3900

.106010

4100

4200

4300

4400

4500

4600

4700

4800

4000 


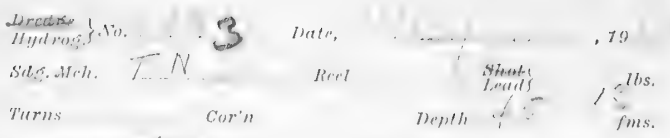

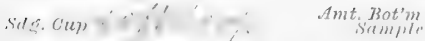

Character of ', , I .

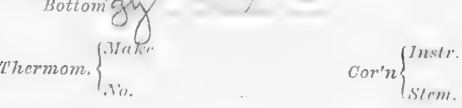

lintlom Reartins

(Cor'd Trmp).

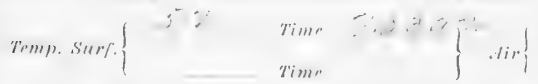

H)insty: Sur\%:

fims.

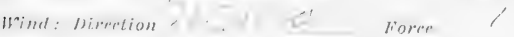

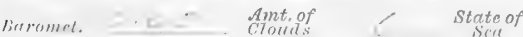

Weatier $\quad c-c$

Direction and Force
of Durrent.

of Ourrent. $\quad$ listlom

Tiule

Generat rocatily

'osition Yaquina Lyeht hears $155^{\circ} \mathrm{E}$ (trac)

chart used: No...6 o. o Kd'n.

Inifl: Dipertion

bistumer

Depth of Haul

Apparatus and Iitig used.

Time lowin

h.

$m$.

liemurtis: 
SOUNIDINR: WIRE.

T.- M. Down.| Un. IT M. $703 \quad 2050$

100)

200

300

400

500

000

700

800

900

1000

1100

1200

1300

1400

1500

titi)

1700

1800

[क(1)

$\because()())$

$\approx 100$

$2: 2()()$

$2: 300$

$\because 100$

$3(10)$

$26(0)$

2700

$: 300$

29)(1)

$: 3000$

3100

$\because \because 30)$

::*ion

$: 100$

$\sin (0)$

: $: 600$

:

$3 \$(0)$

:3900

1000

4100

4200

$4: 300$

$+400$

.1500

4600

4700

4800

4000

DREDIINC (ABI,

M. Dows. IM. $\mathrm{M}$.

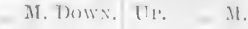




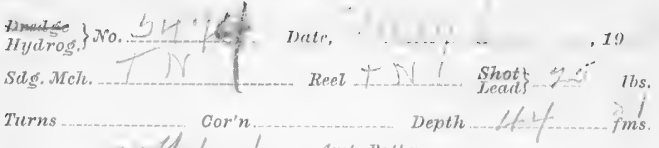

Sas. Oup siffof Amt. Bot'm
Charricter of Bottom

Thermom. $\left\{\begin{array}{l}\text { Mation } \\ \text { No. }\end{array}\right.$ $\operatorname{Cor} n\left\{\begin{array}{l}\operatorname{lns} / \mathrm{r} \\ \text { s/rm }\end{array}\right.$

Borlom liectins

Qur'd Trmp

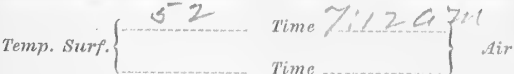

Density: Surp.

Wind: Direction

fims,

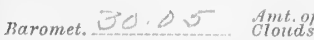

Foref

Weather L $C$

Direction and Force
of Current.

Tikle

General Locality .

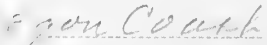

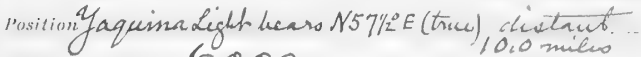
Churt used: No. 6000 Id'n
1)rift: Hirertion
Dislerner

Denth of Haut

Apparatus and Rig zesed

limererlas: 
$11-1023$

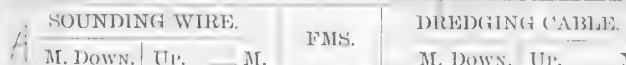

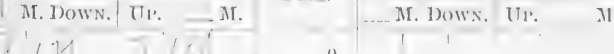

$111+160$

(1)

1(10)

2010

390

100

500

(i)io)

ino

800

(1)

1000

1101

1200

1300

1.400

1500

1000

$1 \% 00$

$1 \times 100$

1900)

2000

2100

2200

2:300

$\because 100$

2500

$\because 600$

$2 \% 00$

asto

2900

:19001

3100

:3:00)

;:301

:3100

(bis)

:460)

:87

:1910

:it)( )

.1000

(11110

1.200

f:1000

1. $1(00)$

1)

$f(i) 0)$

$1 \% 00$

4800

1900 


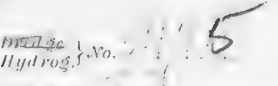

Sels.s. $11 \mathrm{rh}$

Hete,

\section{Turus}

Rin

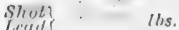

Sag. Cup An 1 . Amt Bont'm.

Character of Bellom

Thermon. $\left\{\begin{array}{l}\text { Wak } \\ \text { No. }\end{array}\right.$

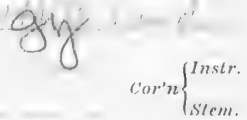

Bottom Reading Cor'd Temp.

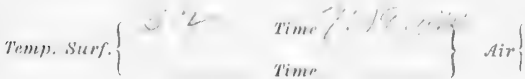

Density: Surf.

fins.

Wind: Direction 1, 22) Force

Baromet. 30.05. Amt.of

State of is

weather $\quad-1:=$

D) irection and Force
of Current. $\left\{\begin{array}{l}\text { Surface } \\ \text { Buttum! }\end{array}\right.$

Tile

General Lormpity

l'osition'y aquina Light bcaroN $607 \mathrm{z}^{\circ} \mathrm{E}$ (Twa) divtant "Hart usest: in. 6000 Vit'l

1)rift: Jirention

Distunew

7)epth of 11 ats

Apparatus and Ris used

limmark:s: 
SOUNDING WIRE.

M. I)OWN. TT. M. $719: 7 \mid 21$
DREDGING CABLE.

FMS. I... M. 1)ow. UH. N.

200

:300)

$4(1)$

.00

(i)

$\% 00$

Si(O)

()()

1000

1100

1201)

13)()

1.100

1500

1(i)

1700

1500

1900

:20)(

2100

200

2)พ00

$210)$

?i, ()0)

: $6(1)$

?ร (1)

sisio)

2) (1)

:Bi)(

2100

$\because 3: 2(1)$

:3:30)

:i10)

:2.)

:itit)

:in)

iston

:

.1001)

.1100

.1?(x)

13060

1100

tiva)

f(si)

17(10)

4800

4900 
IIyil loges on

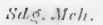

limel i

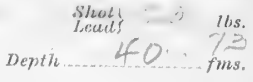

Thros

Cor'n

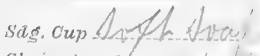
Amt. Hot'm

Oharacter of / Bottom

Thermom. $\left\{\begin{array}{l}\text { Mrak' } \\ \text { No. }\end{array}\right.$

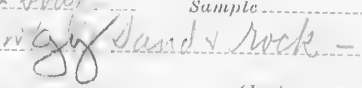
Cor'n $\left\{\begin{array}{l}\text { lnstr. } \\ \text { sitem. }\end{array}\right.$

Bottom Reading

Cor'd Temp.

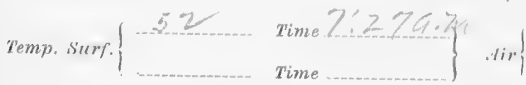

Density: Surf. fins.

Hind: Hirretion Forfe

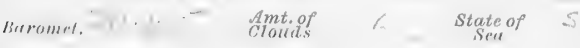
Weather L C $c$

Direction and Force $\left\{\begin{array}{l}\text { Sufure } \\ \text { of Current. } \\ \text { Botlom }\end{array}\right.$

Tille

Generel Lorrlity

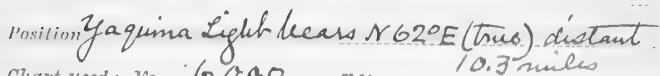
Chart used: Mo. codo nd'n 10.3 ruiles

7)rift: Direction Distance.

Depth of IIaut

Apparatus and libe usei

Remurhas: 
, SUUNDIN(* WIRT.

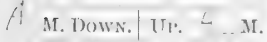

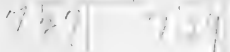

1
liMS.

DREDGTNG CABTF.

M. DOWN, UN, MI.

(1)

200

:30)

.100

5()()

(ii)()

\%01)

Sillo

900

1000

1100

1200

1800

1400

1500

1600

1700

1800

1000

2000

2100

2200

2300

2400

2500

2000

2700

2800

2900

3000

3100

3200

3300

3400

3500

$\$ 100$

:3700

3800

3000

4000

4100

4200

4300

1400

4500

4600

4700

4800

4900 


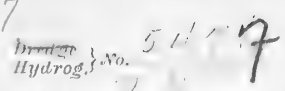
lall",<smiles>C[C@H]1C[C@H]2C[C@H]1C2</smiles>
, $79^{\circ}$

Turus

Cor'n lirel

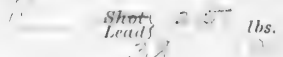

Solv. Cups.

- Depth fms.

(iturartin of

Thermom. $\left\{\begin{array}{l}\text { Malin } \\ \text { Nos. }\end{array}\right.$

Imt. Bot'm

$$
\text { sample }
$$

Botiom Reading

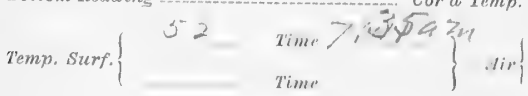

Cor'n $\left\{\begin{array}{l}\text { Instr. } \\ \text { stem. }\end{array}\right.$

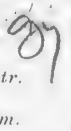

Density: Surf. fms.

Wind: Direction. C L

sinromet, 10 Amt of

$\Rightarrow \quad$ State of

mealluer in at as.

Direction and Horce
of Current. $\left\{\begin{array}{l}\text { Suifuce } \\ \text { Bottom }\end{array}\right.$

Tilde.

Generel horeality

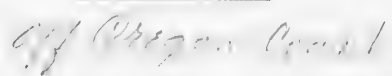

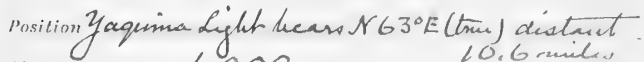

chart used: No .... Go 00 $E d^{\prime} n$

1 Inifl: Dimertion

Distance

Depth of Harel.

Apparatus and Ris used.

Remurk: : 
11- $102: 3$

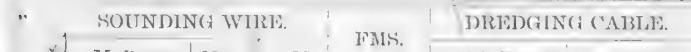

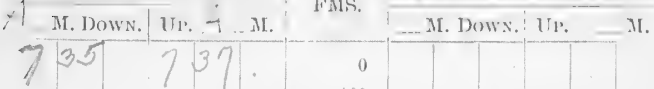

200

300

400

500

000

700

800

900

1000

1100

1200

1800

1400

1500

1000

1700

1800

1900

2000

2100

3200

2300

2400

2500

2000

2700

2800

2900

3000

3100

3200

3300

3400

3500

3600

3700

3800

3900

4000

4100

4200

4300

4400

4500

4600

4700

4800

4000 


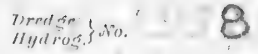

seter. . Helt.

Turns

Cor'n

Sare. Cup:

(huracter of Bollomi

Whermom. $\left\{\begin{array}{l}\text { Huh } \\ \text { ANO. }\end{array}\right.$

Hetr,

, $79^{\cdots}$

Botlom Rearlins

Temp. Surf. $\{52$ linel

$$
\begin{aligned}
& \begin{array}{l}
\text { Shot } \\
\text { beterlt }
\end{array} \\
& \text { gefill }
\end{aligned}
$$
me. Bot'm
Samil,

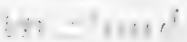

$$
\text { Cor'n }\left\{\begin{array}{l}
\text { Instr } \\
\text { Stem }
\end{array}\right.
$$

Time Cor's Trmp.

Densit!l: Sicref.

fills.

Wind: Direction Cole fore

Baromet. 30,0 Amt.of 2 state of 5

Weather ....... 2

Direction and Force $\{$ Sirfuc"

$$
\text { of Current, } \quad \text { Buttom }
$$

Tide.

Generat Locality Chart used: No... $\mathrm{CO} 00$ Rd'

mrift: Himertion

ristrure

Depth of Ilaul

Apporstas and Rin usat

Time:towins

h.

$m$.

limmelis: 
$11-10=3$

SOUNDING WIRE.
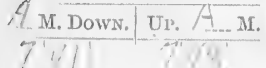

Fas.

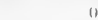

10()

200

:30)

400

500

(3)(i)

\%0()

$\mathrm{s}()()$

(1)(0)

1000)

110)

12(1)

1300

1.100

1500

1 (i)

1700

18iv)

19H)0

2)( $)(1)$

2100

$2+200$

$2: 30(1)$

$\because 100$

$\because 500$

2000

2700

$\because 800$

a)

: $(x)(1)$

3100

:3.: () ()

:3:

:3 (1) ()

3000

:36i(k)

3i(1)

:3(1)

:2nou

10000

$41(1)$

$120(1)$

$4: 300$

1.100

17()()

I $10(0)$

$.1 \%(0)$

1800

4)()()

DREDGING CABLJ:

M.... Down. UTr. .... M. II.
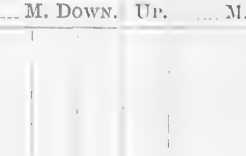


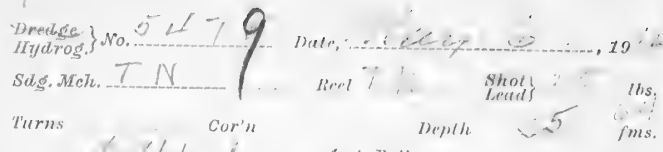

Sas. Cup $\left.x^{\prime}\right)$ Amt, Bot'm
Character of
Bottom

rhermom. $\left\{\begin{array}{l}\text { Make } \\ \text { No. }\end{array} \quad\right.$ Cor'n $\left\{\begin{array}{l}\text { Instr. } \\ \text { stem. }\end{array}\right.$

Bottom Reading Cor'd Temp.

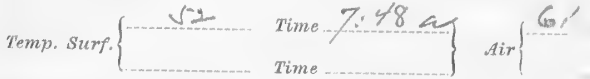

llensity: surf:

fills.

Winil: Direction..... Force

C.?

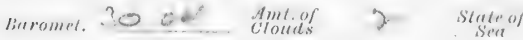

Wealhor



Direction and Force
of Current. $\left\{\begin{array}{l}\text { Surface } \\ \text { lioltom }\end{array}\right.$

Tide

General Locralit!

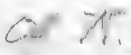

W'

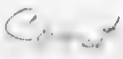

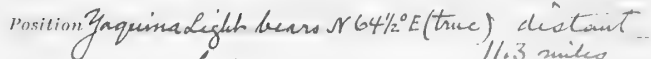
chart used: No,...G.0.0. Fin'n

Drift: Direction

Distunce

1)epth of Haut

Apparatus and Rigused

liemurlis:

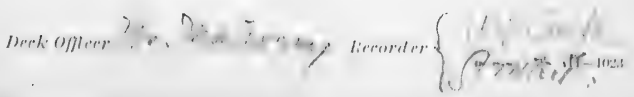


$11-102$

I M. DOW I U A 748
FIIS.

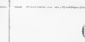

DIREDOINR (ABLE

M. Dow'. ITR. M. 


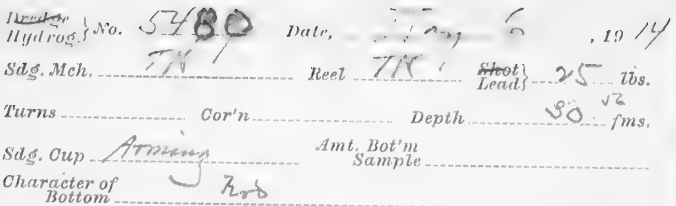

Thermos. $\left\{\begin{array}{l}\text { Male r } \\ \text { A\%. }\end{array}\right.$

Corn $\left\{\begin{array}{l}\text { Instr. } \\ \text { Stem. }\end{array}\right.$

Bottom Reading Cord Temp.

Temp. Surf. $\left.\left\{\begin{array}{l}\text { Time, } \\ \text { Time }\end{array}\right\} \operatorname{Air}\right\}$ ar

Density: Surf.

Wind: Direction fins.

market. sC.

Culotte

Force

(3)

Weather

Ant. of

Q.

State of
Sen

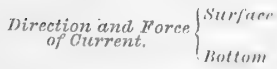

Fid r $\quad \ldots . . .$.

General Locality

tenth Oran cen

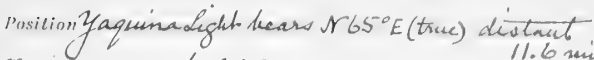
chart used: No. 6000 Eden

11.6 manilas

Drift: Direction

Distance

Depth of Haul

Apparatus and Rig used

Timetorein:

h.

m.

firmerlin:

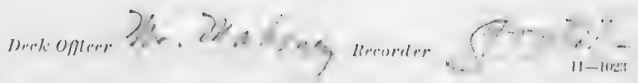


SOUNDING WIRE.

A. M. nown. Iт. Aि. $7 \sqrt{3}$

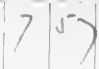

FNS.

DIREDAING ('A13LE.

' M. Dow. ITI. ... M. 


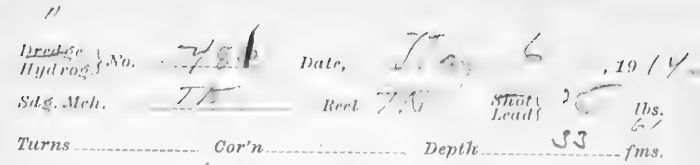

sis. oup Ar... Arming.

Am.t. Bot'm

Churacter of
Botlom

Thermom. $\left\{\begin{array}{l}\text { Makn } \\ \text { No, }\end{array} \quad\right.$ cor'n $\left\{\begin{array}{l}\text { Instr. } \\ \text { Stem. }\end{array}\right.$

Bottoni Reading Cor'd Temp.

Temp. Surf. $\left\{\begin{array}{ll}\sqrt{2} & \text { Tim. } 8.0:-2 \\ \text { Time }\end{array}\right\}$. lir $\}$

Density: Surf.

fms.

Wind: Direction

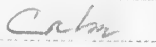

Force

linromet. ? O. o * Antiof

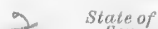

Weather $\chi_{2.1 .}$

Direction and Horce
of Current. $\left\{\begin{array}{l}\text { Surface } \\ \text { Bottom }\end{array}\right.$

Tille.

(inneral loratily

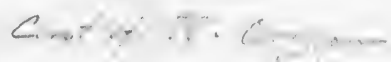

Iosition Yaquins Light hears $N 66^{\circ} \mathrm{E}$ ther) distant-

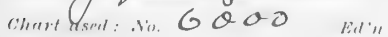

Drift: Direction

Distance

Depth of Haul

Apparatus and Rig used

Time towing

h. $m$.

kPmark:

Trim

8:00 am. rot bulh

83.8 -
Dry hall

87.5 
SOUNDINA WIRE \% Mi Down. Ur. A M. $80 y^{\circ} 84$
FMS.

1)REDAIN(+ ("ABLE.

- M. Dows. Vtr. MI

100

200

:300)

100

500

(i)i)

700

800

$9(0)$

1000

1100

12(1)

1300

1400

1500

$16(6)$

1700

1800

1000

2000

2100

2200

2300

$\because 100$

2500

2600

2700

2800

2900

$: 3000$

$\$ 3100$

:? 00

:i:300

3400

33.00

$\therefore 3600$

$: 3700$

$: 3800$

:300

4000

.1100

1200

1300)

4100

4500

4000

$4 \%(0)$

$4 \times 00$

.1900 


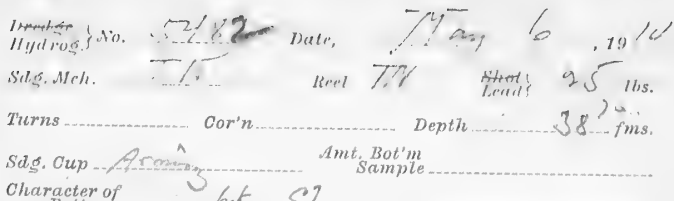

Character of
Bottom 6 ton SR

Thermos. $\left\{\begin{array}{l}\text { Make } \\ \text { No. }\end{array}\right.$ Corn $\left\{\begin{array}{l}\text { Instr. } \\ \text { Sim. }\end{array}\right.$

Bottom Reading

Cor'd Temp.

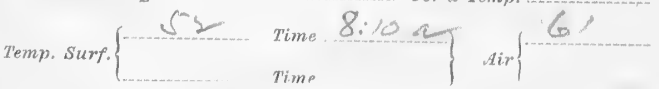

Density: Surf. fins.

Wind: Direction

Carter

Force.

o

Baronet. 30.04 Amt. of Dr State of $S$

weather Es

Direction and Force
of Current. $\left\{\begin{array}{l}\text { Surfers } \\ \text { Bottom. }\end{array}\right.$

Til"

General Locality

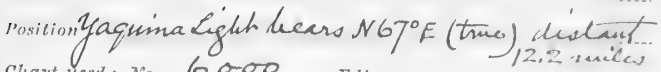
Chart used: No. 6000 Eden

Drift: Direction Distance

Depth of Haul

Apparatus and Ri ङ used

Time towing. h. $m$.

lirmurk's:

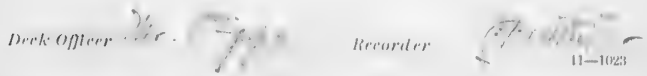


SOLNDING WITR.

$\overbrace{\text { MI. DOWN. UN. }} \pi$ M. 8

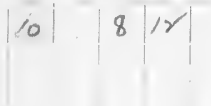

FMS.

DIRTDRING C'ABIAL.

M. Dow: Ur. M. Tr.

100

200

300

$\$ 100$

500

(i00)

700

800

900

1000

1100

1200

1300

1400

1500

16(6)

$1 \% 00$

1800

1900

2000

2100

2200

$3: 3(0)$

2100

2500

2000

2700

2800

2900

$\$ 3000$

3100

3200

3300

3100

3 is 00

:3000

:3700

3800

:900

$+1000$

4100

4: 200

4300

4400

1500

f(ito)

.1700

1800

.1900
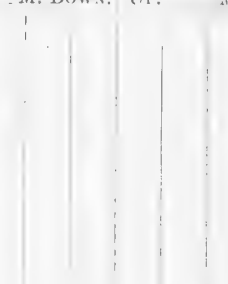


\section{$<13$}

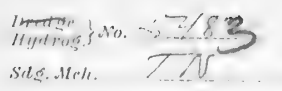

Turns

Cur'n

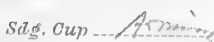

Character of
Bollom

Thermom. $\left\{\begin{array}{l}\text { Mither } \\ \text { No. }\end{array}\right.$

Amt. Bot'm

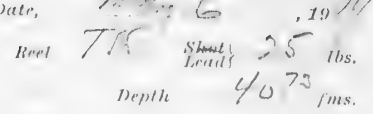
sample

73ottom Reading Cor'n $\left\{\begin{array}{l}\text { Instr. } \\ \text { Stem. }\end{array}\right.$



Density: Surf:

Wind: Direction... $C$ ins:

Baromet. $30.0 \%$ Amtiof 2 state of S

Heather $\mathrm{Cr}_{\mathrm{r}}$

Direction and Force
of Current. $\left\{\begin{array}{l}\text { Surface } \\ \text { Bottom }\end{array}\right.$

Ticto

Ginemal Los:alily

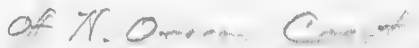

.

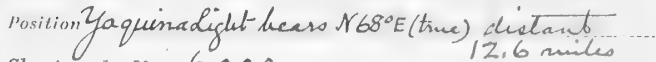
chart used: No. 6000 Ed'n

Drift: Direction Distance

Jepth of Haul

Apparatus and Rig used

limurkes.

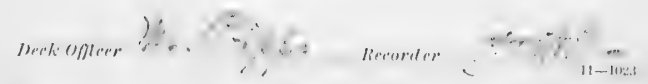


11-1023

SOUNDING WIRE.

FMS.

DREDGING $\div$ ('AILLE

FlM. Down. Ui. A Mr.

$816: 81 / 3010$

M. Dows, IH, M.

100

200

$: 30()$

100

500

(i)

\%0(1)

$\mathrm{s}(0)$

())

1000

1101)

1200

1300)

1.400

1500

1600

1700

1800

1900

2000

$\because 100$

2900

$2: 00$

2100

2500

$\approx 600$

$2 \% 00$

2800

2900

33000

3100

3:00

3300

3400

3.500

$: 3600$

33700

3800

$3: 300$

1000

$\$ 100$

4200

4:300

1400

4500

$+600$

1700

4800

.1900 
14

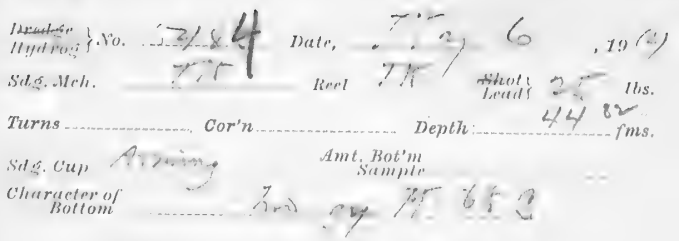

Thermom. $\left\{\begin{array}{l}\text { Nuke } \\ \text { No. }\end{array}\right.$

$$
\text { Corn }\left\{\begin{array}{l}
\text { Instr. } \\
\text { Stem. }
\end{array}\right.
$$

Bottom Reading Cor'd Temp.

Temp. Surf. \{ Time 8.27 a $\}$ Air $\{$ 6

Density: Surf. fms.

Wind: Direction Cue bor.., For re

Baronet, 3 Q $0 \&$ Amt of State of

Weather

Direction and Force
of Current. $\left\{\begin{array}{l}\text { Sulfur" } \\ \text { Bottom, }\end{array}\right.$

Tide

General Locality

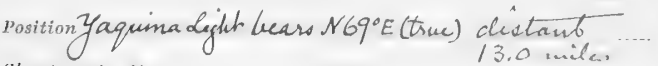
chert used: . Foes.

EN"

Drift: Direction

Distance

Depth of Haul

Apparatus and Rig used.

Time toxins

li. $m$.

limbers:

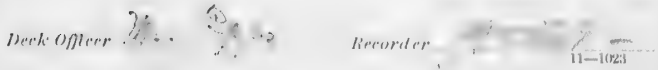


$11-1023$

\section{SOUNDING WIRE.}

A M. Down. U1. A M. 827

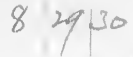

FMS.

DREDGING CABLE.

.... II. DOWX, U1. ... M.

()

100

200

$\$ 300$

400

500

000

i()(i)

800

900

1000

1100

1200

1800

1400

1600

1600

1700

1800

1000

2000

2100

2200

2900

2400

2500

2600

2700

2800

2900

3000

$\$ 3100$

3200

33:30n

3400

3500

$\$ 300$

3700

$\$ 800$

3900

.1000

4100

4200

4300

4400

4500

4000

4700

4800

4900 


\section{5}

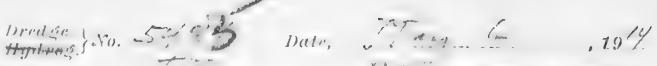

Sids overte.

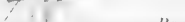

$\because$

Shet

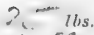

$6 \%$. 19

Turms

Cor'n

l)epth

fins.

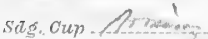
Amt. Bot'm

character of Boltom

$$
\text { Sample }
$$

Thermom. $\left\{\begin{array}{l}\text { Hul: } \\ \text { No. }\end{array}\right.$

Cor'n $\left\{\begin{array}{l}\text { Instr. } \\ \text { Stem. }\end{array}\right.$

Bottom Readins

Cor'd Temp

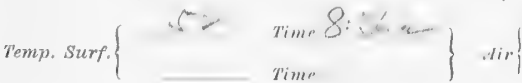

.

Deusity: Surf.

Wind: Jirection

Foree

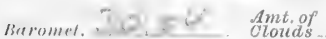

State of

Weather t...... Z

Direetion and Force
of Current. $\left\{\begin{array}{l}\text { Surfure } \\ \text { Jisltom }\end{array}\right.$

Tirln

Generrel Loratity

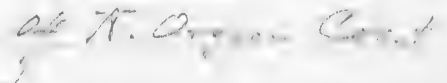
;

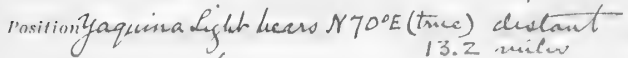
ohart usnd: No. Go.00 Indn

Hrifl: llirerlion

Dis/rulle"

Depth of Haut.

Apparatus and ligi used ...

Timetouins

l.

$m$.

limmarles: 
$11-1023$

SOUNDING WIRE.

A M. Dows. U1. A . M. $8|36: \quad 8| 39 \mid$
FNS.

DREDGING CABLE.

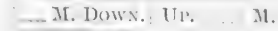

100

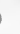

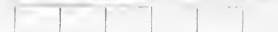

200

300

$9(10)$

500

(ii)

\%01)

$800)$

(x)

1000

1100

1200

1906

1.100

1500

1600

1700

1800

1900

:2000)

$\because 100$

2000

$2: 300$

$\because 100$

2300

$\because 600$

จ

ख्या)

39000

$: 3(900)$

$\$ 3100$

:3:190

:3:3001

:3100)

3ision)

:(6)

$: 1 \% 00$

: 3000

$\$ 100$

(1)(1)

1100

1200

1300

1400

4.

$4000)$

1700

|

.1900 
$16-\therefore$

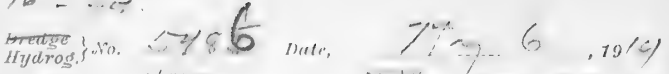
Sols. Mr h. Z Z Turns Corn kind lis.

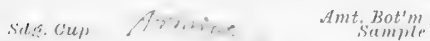
Character of fro $b$ ic $\hat{\varepsilon}$ thermom, $\left\{\begin{array}{l}\text { Make } \\ \text { Sis. }\end{array}\right.$ Corn $\left\{\begin{array}{l}\text { Instr. } \\ \text { Stem. }\end{array}\right.$

Bottom Reading Cord Temp.

Temp. Surf. \{ i: $\quad \operatorname{Tim} \overline{\bar{S}} \cdot \overline{9}$

Density: Surf

Time

ar $\operatorname{sir}$ !

(.)

Wind: Direction

intromit.

So Ant. of

(s)

Weather

thing

1) direction and Force
of Current.

Titer

General Loralit!l Oaf?

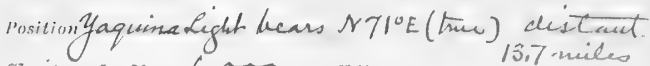

Char it used: No

GooD Ind n

mInify: Direction

Disterture

Depth of Hart

Apparatus and Rig used

Time towing

h.

$m$.

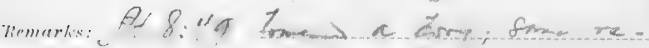

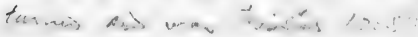

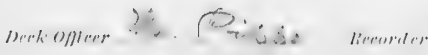

$\therefore \quad \because \frac{1}{11-102}$ 
$11-1023$

$\rightarrow$ SOUNDING WIRE.

$\because$ M. DOWN.|Vir. MT.

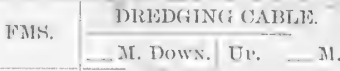

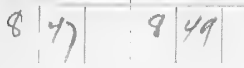

(on

$2(1)$

300

$4(k)$

500

(i) (i)

;i)()

800

(HoO

100()

$110 t$

1200

1:30)

1.100

1500)

1(i) ()

170()

18() 0

1940

$\because()() 0$

2100

221) 0()

20)

$\$ 100$

2500

2000

2700

2800

?)

:i) (1)0

$: 3100$

:is)(

:3i)

$\because 100$

300()

: ilike

:บ1)

:3(1)

:inou)

. $1(0)()$

(11)( )

(130)

. 1:i0)

110()

1.iv)

Ifioc)

.1701

4800

.1900 
$M-S_{e t}$

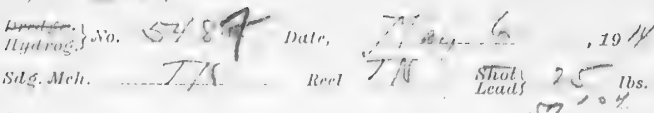

Turns Cor'n Depth 17 fms. Sas.Cup Atrrmins Amt Bot'm Character of EXS GES $S 7$

Thermom. $\left\{\begin{array}{l}\text { Make } \\ \text { ro. }\end{array}\right.$ Cor'n $\left\{\begin{array}{l}\text { Instr. } \\ \text { siem. }\end{array}\right.$

Bottom Reading Cor'd Temp. Temp. Surf. $\left\{\begin{array}{c}52 \\ -12\end{array}\right.$ Time $8 . \sqrt{3}$ Time $\operatorname{Air}\{6\}$

Densily: surf.

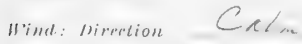
fims.

Buromet. ?O, \&. Amt of

c) Weather State of Direction and Force
of Current. $\left\{\begin{array}{l}\text { Surfuef } \\ \text { Bottom }\end{array}\right.$ Tille

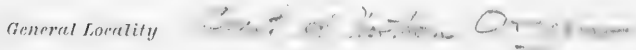

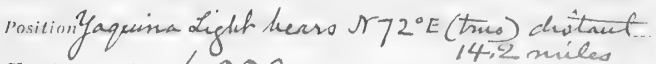
chart used: No 6000 Tith 1mifl: Dirertion Distaner Depth of Haul Apparatus and Kis nesed ...

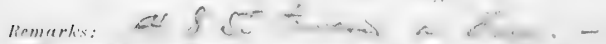

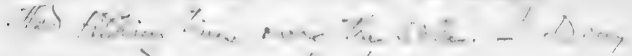

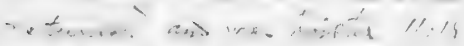
Pottom Simples vas at 11:15

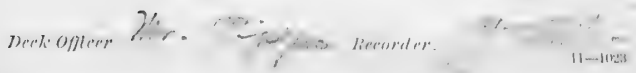




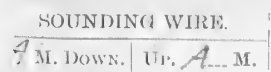

FMS.

DREDGING CA7BLE.
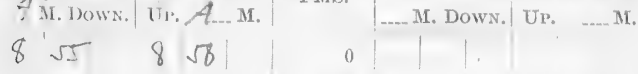

2100

$2: 00$

2390()

94100

2500

$2(0)$

2700

28:(1)

23:00)

$\because(0) 00$

$\$ 100$

:3200

:.:300

3100

$\therefore(0)$

:300

:"700

:350)

$: 300$

.10000

.1100

.1200

$4: 300$

(1) 100

15001

If(60)

(7)

|1500

4906 


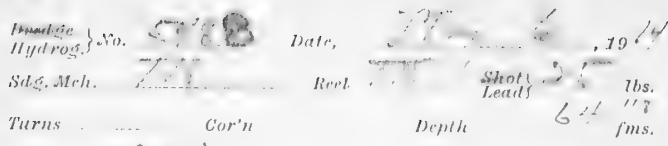

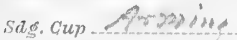
Amt, Bot'm
Sample

Character of Bottom $2 x 8$

Thermom. $\left\{\begin{array}{l}\text { Make ................ } \\ \text { Nir. }\end{array}\right.$ Cor'n $\begin{array}{l}\text { Instr. } \\ \text { Stem. }\end{array}$

Bottom Reading Cor'd Temp.

Temp. Surf: $\left\{\begin{array}{ll}\sqrt{2} & \text { Time } 1 \% \omega 0 \\ \text { Time }\end{array}\right\} \operatorname{Air}\{ \pm ?$

Dersit!l: Surf.

fins.

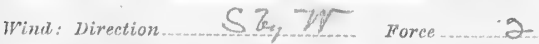

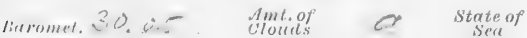

weather ...... to

Direction and Foree
of Durrent. $\left\{\begin{array}{l}\text { Surtum } \\ \text { Botlom }\end{array}\right.$

Tillo

\section{General Loratity}

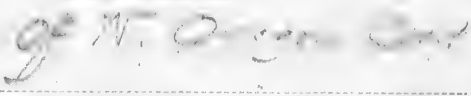

Insilion'yaguina Light heare N75\% E (truc) distrust 14.1. chart used: No. 6000 IEd'n

Drift: Direction Distance

1)epth of Haut

Apparatus and Rig used.

liemertis: 


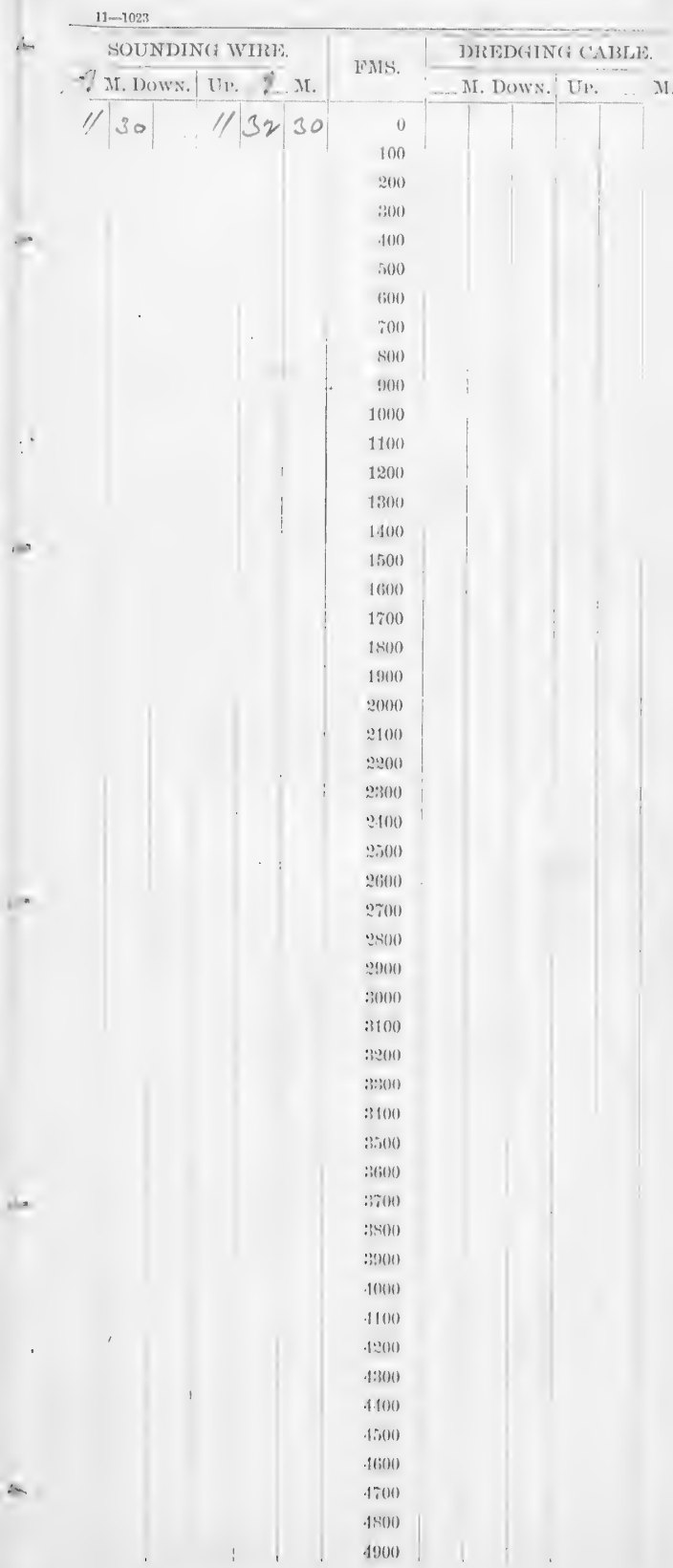




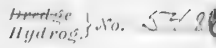

Sals. $\mathrm{Mc} /$ 72

I) (1)

$-r i n$

$\therefore \quad, 19 \%$

Turns

$C\left(1 r^{\prime}\right)$

$y \cdot i$

$\left.\begin{array}{l}\text { Shont } \\ \text { lecal }\end{array}\right\}$ los.

Je)ilh

6,112

sag. oup _. Aruzing Amt. Bot'm

Character of
Bottom

Thermom. $\left\{\begin{array}{l}\text { Make ............... } \\ \text { No. }\end{array}\right.$ Cor'n

Bottom Reading

Cor'd Temp.

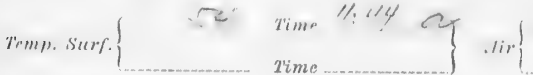

Inusity: Surf.

fins.

Wind: Direction . S

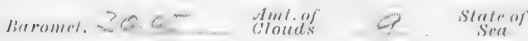

Ifrealher

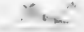

Direction and Foree $\left\{\begin{array}{l}\text { Surfaen } \\ \text { of Current. }\end{array}\right.$

Tider

General Loicalil!

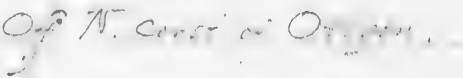

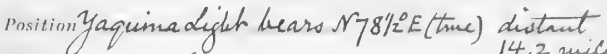

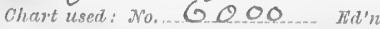

1)rift: Dirertionn

Jisture.

Depth of Haut

Apparatus and Rig used

lienurelis:

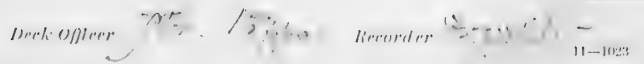




\section{SOUNIDIN(}

? M. Down. U1. M. M.

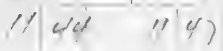

FMS.

DILDGING CABLE.

1)

AI. Dow. II. II.

100

200

:3() ()

$100)$

500

(i) 0

\%00

$8(10)$

(1)(1)

1000

1100

$1: 200$

1300

1900

1500

1600

1700

1800

1900

2000

2100

$\stackrel{2}{200}$

2:30)

2100

2500

2000

2700

2800

2900

$: 3000$

3100

:3200

:3isoo

3100

3500

:31:00

$: 3700$

$3 \$ 500$

3900

.1000

.1100

1200

4:30)

4.400

$45(1)$

4600

4700

1800

(1900) 


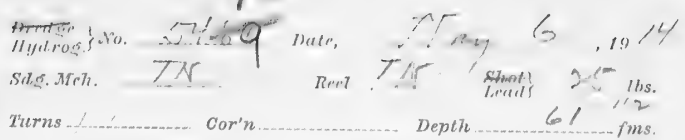

sas. Cup ... Lirminy Amt. Bot'm

Character of
Boltom

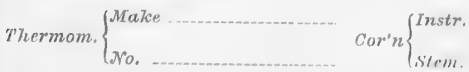

Bottom Reading Cor'd Temp.

Temp. Surf. \{

rime

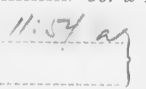

$\operatorname{sir}\{\sin 2 \cdot 50$

Density: Surf.

fins.

Mint: Hirretion

Baromet. $30.0 \mathrm{~s}$

Fore: 2

reather 10 .

Direction and Force
of Current. $\left\{\begin{array}{l}\text { Swriter } \\ \text { Diollom }\end{array}\right.$

Tilln

(imnerret Lorrtily

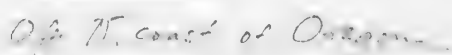
Amt.of 0 State of

PositionYaquina Light hears $181^{\circ} \mathrm{E}$ (truc) ditant... chart used: No. GOOO.. Jin

Drift: Direction

Distanee.

Depth of Haul

Apparatus and Rig used

Time towing h. $m$.

Remarks: 
$11-1023$

SOUNDING WIRE.

Q. M. Down. UP, Q.M. $1 / 54 \quad / 15600$
FMS.

DREDGING CABLE.

…M. Down, UP. .... M.

1010

200

:00)

100

500

(30)

70)

solo

(1)0

1000

1100

1200

$180)$

1400

1501)

16000

$1 \% 00$

1800

1900

$\because 000$

$\approx 100$

200

2330

2400

$\therefore 500$

2006

$2 \pi 00$

2800

2900

3000

3100

: $: 00$

:3:300

3500

3500

3610

3200

:3800

:3901

4000

$\$ 100$

4200

(1:30)

4.400

4500

4600

4700

1800

4900 


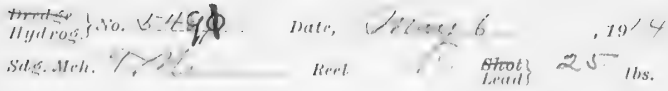

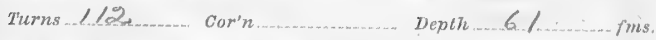

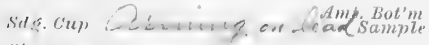

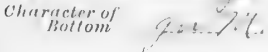

Thermom. $\left\{\begin{array}{l}\text { Make ........................ } \\ \text { No. }\end{array}\right.$ Cor'n $\left\{\begin{array}{l}\text { Instr. } \\ \text { Sirm. }\end{array}\right.$

Bottom Reading ........................ Cor'd Temp.

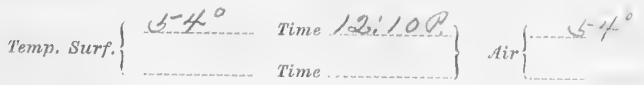

Density: Surf.

fins.

Wind: Direction.. P.X. Force 2-3

Baromet, 3 0. S- Amt of

Weather iffe.

State of

Direction and Floree
of Current. $\left\{\begin{array}{l}\text { Surfar } \\ \text { Bottom! }\end{array}\right.$

Tide

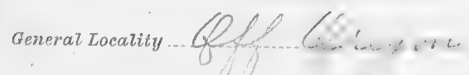

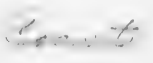

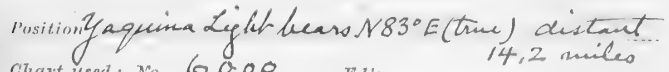
chart used: No. G0.00 $E^{\prime}+l^{\prime} n$

Drift: Direction

Distanse.

Depth of Haul

Apparatus and Rig used

l'immturius.

fi.

$m$.

IIPMUルk: 
SUUNDING WIRE.

$P$ M. Jown. Ur. 70 M. $121070012 \log 130$
DIREMING (CA1BT,

M. 
22

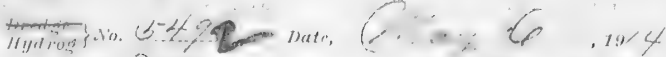

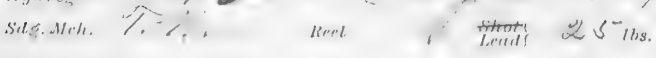

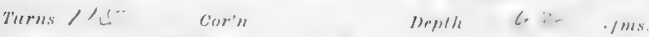

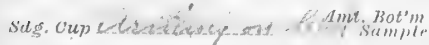
Character of
Botlom

Thermom. $\left\{\begin{array}{l}\text { Make............. } \\ \text { No. }\end{array}\right.$ Cor' $\begin{array}{l}\text { Instr. } \\ \text { Strm. }\end{array}$

Bottom Reading Cor'd Temp.



Density: Surf. fms,

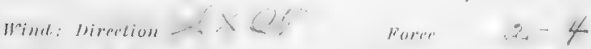

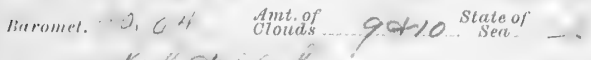
Weather Hey of

Direction and Force
of Current. $\left\{\begin{array}{l}\text { Surfur } \\ \text { Bottom. }\end{array}\right.$

Tiler

Genlmal loratit!

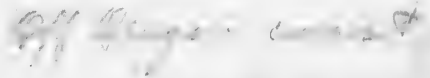

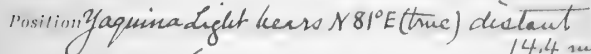
chart userl: No. 6000 Ind'n 14.4 mile, Drift: Direction

Distance

Depth of Haul

Apparatus and Ris used.

Time towing h. m.

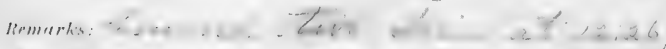

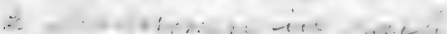

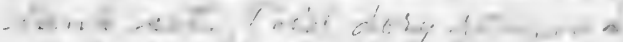

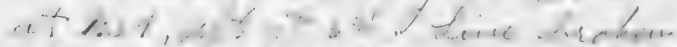

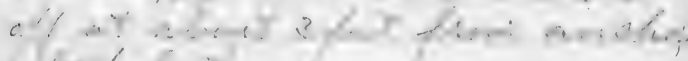
- . 1 .

Tine lio P.2n. Wet bule Dry bull

$5 \% .4$ 5 is. 0

berk offur. tis : c....

limererter

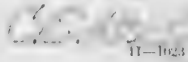


11-1023

SOUNDING WIRE. NM. Down. Ur. U.

$12+260012=2+60$

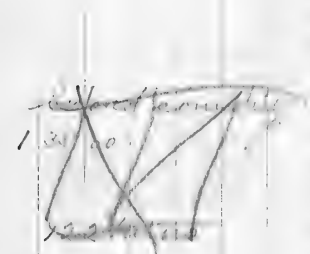

1000

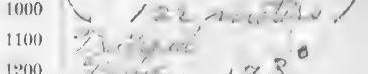

1200

1300

1.100

$1500)$

I(300)

1700

1800

1000

$20(0)$

2100

2301

$2: 3(k)$

$: 400$

$\therefore 500$

2600

2700

2800

2900

3000

3100

$\$ 200$

$: 3300$

$\$ 3100$

3500

3660

$: 3 \%(0)$

3800

3900

.1000

1100

4200

.1300

4100

.1500

1600

.1700

1800

4900

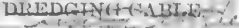

M. Dow. Tir. J $M$.

1 ! 1000,19600

, $14.5 \%$

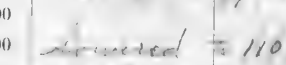

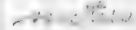
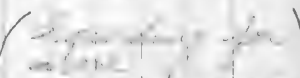

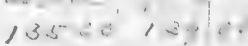

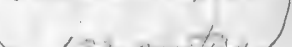

$\because 7, \quad, 3$

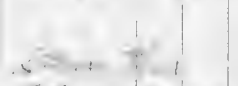

$r \therefore i \quad ! 0:-1, \ldots$ $-4 \%(2)$

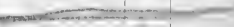

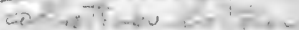

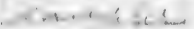

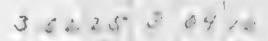




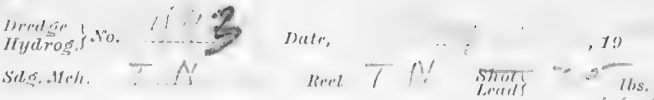

$$
\begin{aligned}
& \text { Turns } \\
& \text { Cor'n } \\
& \text { Depth } 76 \text {. } 15
\end{aligned}
$$

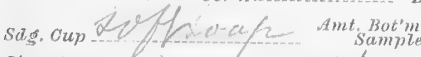

Character of liottom

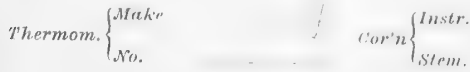

Bottom Reading

Cor'd Temp.

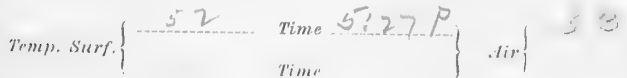

Density: Surf. fins.

Hinde: Himetion

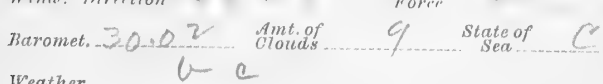

Weather

$$
\text { Direction and Force }\left\{\begin{array}{l}
\text { Surfure } \\
\text { of Current. }
\end{array}\right.
$$

Tile

Coneral Locality

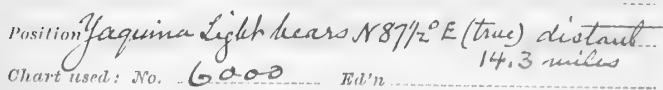

Jrift: Direction

Distance.

Depth of Haut

Apparatus and Ris used

liemurks: 


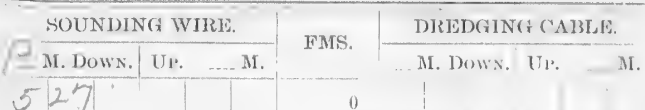

\begin{tabular}{r|r|r}
527 & 0 \\
& &
\end{tabular}

200

:300

100

500

(it)o

500

8(1)

(9)0

1000

1100

1:(0)

1800

14110

1500

16(6)

1700

1800

1900)

2000

2100

2200

2:300

3100

(500)

$2(i 0)$

2ริตก

उ800

2900

$\$ 3000$

$\$ 100$

3:00

3300

3400

3500

$: 0000$

:3100

3800

3900

.1000

$\$ 100$

1200

4300

4400

4500

$\$ 600$

1500

4800

4000 


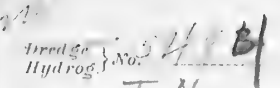
Sas.Mch. T N

Dute, $i 1 \leqslant \div$, Reel $7 \mathrm{~N}$

Shot

Turns Cor'n.

Depth 72

fins.

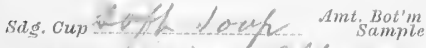

character of Botlom

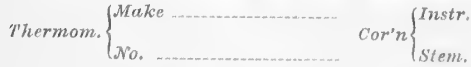

Bottom Reading Cor'd Temp.

Timm. Surf. $\left\{\begin{array}{l}\text { Time. } \\ \text { Time }\end{array}\right\}$ Air $\}$

Density: Surf. fins.

Wind: Direction... SE 55 Horce 2 Baromet. 30,02 Ant of Clowds 2 State of

\section{Ifrather}

Direction and Force
of Ourrent.

Tid"

(irneral Lorrlil

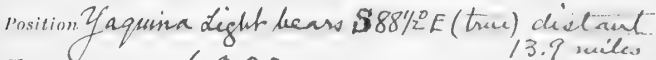
chart used: No, 6.0.0.0... Henn Inrift: Mirerlion

Distantre

Depth of Haul.

Apparatus and Rigused $m$

Nemarks : 
SOUNDING WIRE: T. DOWN. TH. .... M. M.

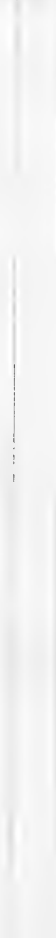

DREDGING GABLE.

FNis.

-... M. Down.| UI. _ M,

1)

(100)

20)

:30)

100

500

(i)(i)

$\pi 00$

Si)(

()()()

1000

1100

12001

130)

1.400

1500

$1(i) 0$

1700

1 sisto

1900

$2(30)$

2100

$20(0)$

$2: 300$

2100

2500

2600

$2 \%$

2000

2900

$: 000$

$:: 100$

:3200

;i:ivor

:31(k)

:3500

:300)

:300)

$39(x)$

$: 3901$

10)(1)

1100

1200

4: 1800

$+100$

4.500

4600

(i⿱宀⿻)丨

स:(10)

+1000 


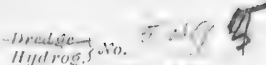

liate

sids. derth. $>$ 入

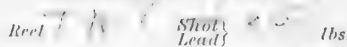

Turns

cor'n

Depllte

fims.

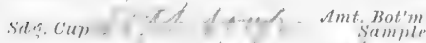

Wharacter of
Bottom

whermom. $\left\{\begin{array}{l}\text { Mrther } \\ \text { Wo. }\end{array} \quad\right.$ Cor'n $\left\{\begin{array}{l}\text { Instr. } \\ \text { stem. }\end{array}\right.$

Bottom Reading Cor'd Temp.

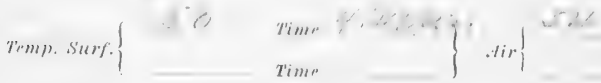

Mensity: surf: fims.

Wind: Direction E $\quad$ Foree 2

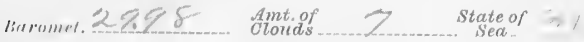

weather l... C

Direction and Force
of Current.

Tilde

Gen"rel Loprlily

'osilion Yaquina Light bears $885 \% 2^{\circ} \mathrm{E}$ (true) distant chart used: No 6000 man

Irrifl: Mirertion

Distaner

Depth of llaut

Apparatus and Rig used.....

Time towing

h.

$m$.

Remurles: 
to SOUNDING WIIRE.

7 M. Dow: Us. _ I.

$41 \therefore$
FMS.

DIRTDGING CABLE.

\begin{tabular}{l|l}
.... M. Down. Ur. …. M. \\
\hline
\end{tabular}

201)

:30)

10)

i $(00)$

(i) (1)

ร0)

$800)$

(1)()

1000

11 (l)

1:(3)

$120(1)$

1400

1500

I(6)()

$1 \% 00$

$1 \mathrm{~s}(0)$

1900

20000

2110

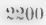

2300

3400

2500

2(i)0

$2 \% 00$

ㅁ900

2900

: $: 001)$

$\$ 3100$

:खeo

:2:300

$\$ 100$

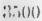

:)(ion)

:สัต⿰)

s. 400

:3900

(1001)

4100

1200

4300

4400

.1500

(160)

(1)

1500

f(90) 

$11-1023$

SOUNDING WIRE.

7 M. Down. Ur. .... M.

Gremdininit cantal.

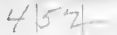

Fals.

M. Down. UP. M.

1

100

200

$3(0)$

$+100$

, 100

(i)

T(O)

$8(0)$

()00)

1000

1100

$12(0)$

$120)$

1.100

1500

$1(6) 0$

1700

1800

1900

2900

$\approx 100$

200

$2: 300$

$\because 190$

2500

2600

25110

2क्ष(1)

บ?ต(1)

$: 1000$

$\$ 3100$

:39(x)

:3:3)

3100

$33(10)$

:3tion

:3i(1)1

:84t10

:ii)()

(10)10

$41(1)$

4200

4300

1400

$\sin (00)$

4600

1700

(1500

.1900 


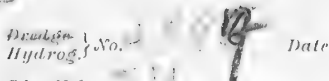

Suls.Alrh.

Turns

Cor'n

sas. Cup ofoth dotef. Amt. Botm

Charqcter of
Bottom

Thermom. $\left\{\begin{array}{l}\text { Hukn } \\ \text { No. }\end{array}\right.$

Cor'n $\left\{\begin{array}{l}\text { Instr. } \\ \text { stem. }\end{array}\right.$

$\left.\begin{array}{c}\text { Shot } \\ \text { Letedi }\end{array}\right\}, 19$

Deplh

fims.

Ballom Rerulins

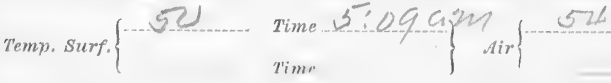

Density: Surf:

Wind: Direction E

fms.

Buromet. $\because \cdots \quad \begin{gathered}\text { Amloof } \\ \text { Eloudes }\end{gathered}$

Force $\frac{2}{\text { State of }}$

Wralher

n $\quad \ldots+\ldots$

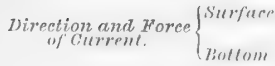

Till"

Generel Lomlity

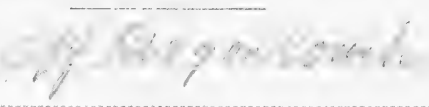

Position Yaquina Light hears $576 \%$ E (twe), diatant

Chart used: No, 6000 Ed'n

Inift: Disertion

Disturuen

Depth of Haut

Apparatus and Ries used.

Time towing

h. $m$.

limmers: 
$11-16: 9$

. SOUNDING WIRE.

f. M. JOWN.|Ur.' M.

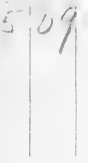

FMS.

FIREDGINA (ADLE.

... M. 1)0w. II. .. M. ()

100

20()

300

10)(

500

(it)

700

800

8()()

1000

1100

1i())

1301

1400

1500

16(i)

1700

1800

19)()

2000

"10)

2खO

2):

2100

35(0)

s(iv)

$2 \pi 0(0)$

जरा11)

s(n)()

:Bकolo

:3100

:3)( )

:3ison

:11)

䙵)

:n(i)

:3)

:3sos)

:35)

.10000

I $1(x)$

1200

.1300

4.900

1.50)

1000

.1700

4800

.1900 


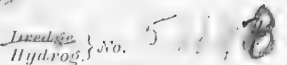

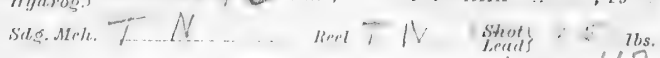

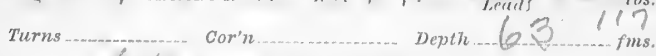

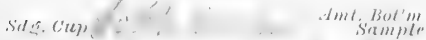

Character of
Bollom

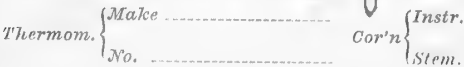

Botlom Riralins

Cor'rl T'rmp.

Trmp. Surf. $\left\{\begin{array}{l}\text { Time } \because(j) \\ \text { Time }\end{array}\right\}$ tir $\}$

Density: Surf. fims.

Wind: Direction...... 2

liaromet.

$\because 2, \% 8$

Ant. of Force

Weather i.) $: 5-7$

State of Ser.

1)irection and Force
of Current.

Tider

General Loertlit!!

Position'Jaquinadylch hears $572^{\circ} \mathrm{E}$ (trive) clietrut..

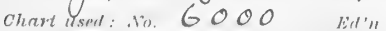

Drift : Direction

Distance

Depth of Haul

Apparatus and Rig zcsed.

dirmarls: 
$11-163 n$

SOUNDING WIRE.

¿f M. Down. UP. _... M.

IDRTDGING C $\triangle \mathrm{BIJ}$

$5730 \quad 7 \div:$

M. Dowx. Hก. M.

I(1)

20()

$: 2()()$

(10)?

500

(in)

700

800

9)(

1000

1100

1200)

1800

1400

1500)

1600

1700

1800

1900

2000

2100

2200

$2: 300$

$\because 1+4)$

2) (10)

$20(0)$

พั)

300

2)(1)

:3000

:3100

:3) 00

:3 300

:3100

:Bi)

:ili)

$\because 8(1)$

:38(4)

:1)(0)

.10010

(11)

1.2010

1:300

4100

4500

dito()

fi(t)

Asina

.1900 
Himedise
Hiflios,

Sils. Atrh. … O?

I'urus Surus. Cup Ler Cor'n rule,

Siml

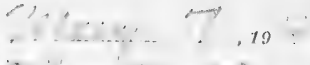

Shoot
leculs $\quad \because \cdot$ ths. 1)epith

$\therefore \therefore$ fims. lsollom

thermom, $\left\{\begin{array}{l}\text { Nulie } \\ \text { No. }\end{array}\right.$

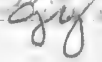

Amt. Bot'm

Bottom Reading Cor'n $\left\{\begin{array}{l}\text { Instr. } \\ \text { stem. }\end{array}\right.$
Temp. Surf.
$(\because ;)$

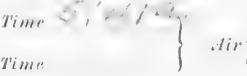

Cor'd Temp.

Density: Surf.

Wint: Nirection

lioromet, $=2,9$ Amt.of

Healier \& $\$$ fins.

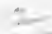

strute of Set

Fore

$\div$

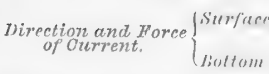

Tillr. .

General Locratity

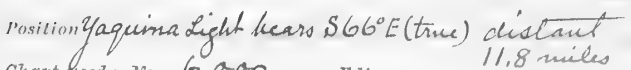

chart thed: No, Gado $H^{\prime} d d^{\prime}$ 11,8 miles

1).ifl: Nirertion

Distume

Depth of Haut

Apparatus and Rig used

diemertio: 
SOUNDING $*$ WIRE.

Ar. Down. Ur. Tr.

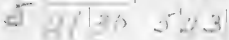

INMS.

DRFDAIN ( CABLA:

_. M. Down. Un. .. II.

100

200

:300

400

500

(it)0

700)

$8(1)$

)(1)0

10)0

1100

1200

1300

1.400

1500

1600

1700

1800

1900

2000

2100

$2: 00$

$2: 300$

2400

2500

2600

27(00)

2800

2900

8000

$\$ 100$

$: 3200$

83800

3400

3500

:6600

$8 \% 00$

3800

:3900

.1000

4100

.200

4300

4400

4500

4600

4700

IN00

.1900 
6

Drratser

llentrostato.

Jate.

Selg. Mch.

Reel

$1 !$

Turns $\operatorname{Cor}^{\prime} n$ Depth Shot\}, of

Sass. Cul :

chreructer of

Thermom. $\left\{\begin{array}{l}\text { Make } \\ \text { No. }\end{array} \quad\right.$ Cor'n $\left\{\begin{array}{l}\text { Instr. } \\ \text { Stem. }\end{array}\right.$

Bottom Reading. Cor'a Temp.

Temp. Surf. $\left\{\begin{array}{l}\ldots \\ \ldots\end{array}\right.$

Time Time fins.

Density: Surf.

rins.

Wind: Direction.... 5

Force 42

liaromet, $32, ? 5$

Amt. of

ay

State of
Sra

Weether

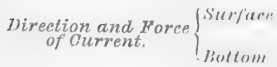

Tiate

Generat Locality

YCosegan Crarh

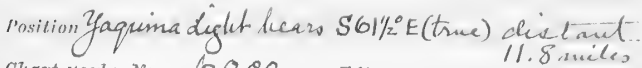
chart used: No. SOOQ0 E'l'n

I)rift: Hirention

Distaturn

Depth of Haul

Apparaius and Ris used.

fimetomint

h.

$m$.

lipmitrk:

Tim

$6: 000.84$. rret bueh Dry buth

50.5

$5 \div 0$

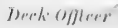

lierouder

$11-112 \pi$ 
SOUNIINA WIRI.

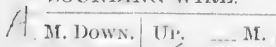

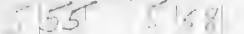

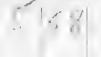

FMS.

. M. Dow: H1, M.

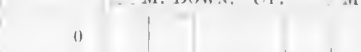

200

$: 300$

$\$ 00$

500

600

700

800

()( ) ()

$10(1) 0$

1100

1200

1300

14100

1500

16000

1700

1800

1900)

2000

2100)

2.200

2300

$2 \cdot 100$

2500

2600

2700

2800

2900

$: 3000$

3100

$: 3200$

3300

$\$ 100$

$\sin 00$

$: 3600$

:3\%0

$: 3800$

$: 3900$

.1000

1100

4200

4300

4100

.1500

4600

4700

4800

f(n)(x) 


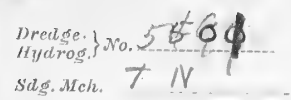

Date, ...', T N 1012 Reel T/N strots 2 ills.

Turns

Cor'n.

Depth $\&$ \%

(i)

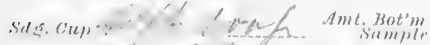
Character of ive
Bottom

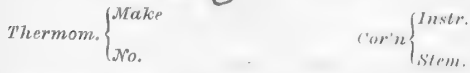

Bollom lituding Cor'd Temp.

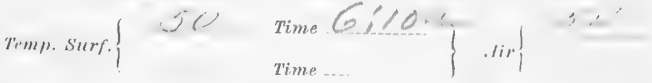

Densily: Surf.

Wind: Direction.

Baromet. 20.75 Amt of $\rightarrow$ State of

weather. \& $6>1$,

Direction and Force $\left\{\begin{array}{l}\text { Surfus" } \\ \text { of Gottom }\end{array}\right.$

Tide

General Locality $C$

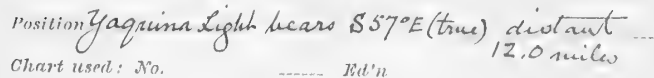

lut'n

Drift: Direction

1)istance....

Depth of Hatet

Apparatus and Ris usert.

Remarks : 
SOUNDINA WIRE.

Y7. M, DOWN.| U1, Y7. M. $6|10| \because 6|/ 3| 0$

100

200

:30)

400

500

(i)

700

8() 0

(9)

1000

1100

1200

1300

1400

1500

$10(1) 0$

1700

1800

1900

2000

2100

2200

2300

2400

2500

2600

2700

2800

2900

$: 3000$

3100

$: 3: 00$

:3300

3100

3500

$: 3600$

:ะช 00

3800

3900

.1000

.1100

1200

4300

4400

4500

4600

.1700

.1800

4900
DREDGING CABLE.

..... Mr. Down, UP. .... M. 


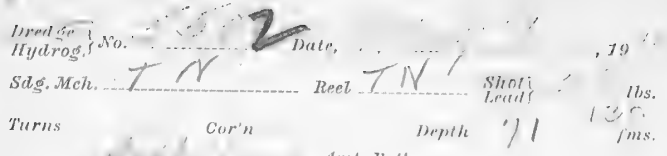

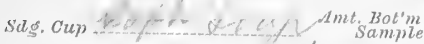

Character of
Bottom

Thermom. $\left\{\begin{array}{l}\text { Makte .................... } \\ \text { Nor. }\end{array}\left\{\begin{array}{l}\text { Instr. } \\ \text { Strm. }\end{array}\right.\right.$

Bottom Reading Cor'd Temp.

Temp. Surf. $\left.\left\{\begin{array}{l}\text { Time } \\ \text { Time }\end{array}\right\} \operatorname{Air}\right\}$

Density: Sturf.

fins.

Wind: Direction . E

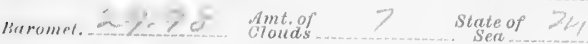

Weather _..... C $\quad 271$

Direction and Force
of Current. $\left\{\begin{array}{l}\text { Surfuen } \\ \text { Bottom }\end{array}\right.$

Tile

General Locality

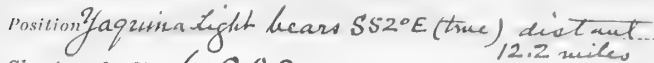
Chart usert: No. GOOO Zl'n

Drift: Direction

Distance

Jenth of Haut

Apparatus and Rig used.

h. m.

Remarlis: 
SOUNDING WIRE.

Af. M. Down. UP. T. M. $6 \sqrt{25} 625$
FMS.

DIREDGING $\div$ CAI3LE.

M. Down. Ur. ... Mr.

100

200

300

400

500

600

700

800

900

1000

1100

1200

1300

1400

1500

1600

1700

1800

1900

2000

2100

2200

2300

2400

2500

2600

2700

2800

2900

3000

3100

3200

8300

3400

3500

$\$ 3000$

3700

3800

3900

4000

4100

4200

4300

4400

4500

4000

4700

4800

4900 


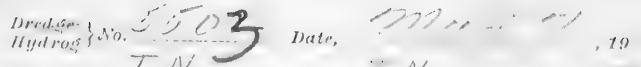

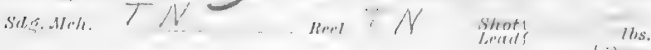

$$
\begin{aligned}
& \text { Turns_.... Cor'n...... Depth -7/ } 130
\end{aligned}
$$

Botlom Rearling Correl. Trmp.

Temp. Surf. $\{\quad \therefore i$,

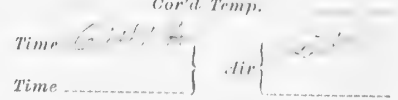

Hensily: Surf.

Wind: Direction $S E \times E$ fims.

fircromet. $\therefore 7$ \& Mint of

Weather ........ $\mathrm{C} z \mathrm{~m}$
Direction and Force f Shrier of Current. $\{$ Bottom

Till,

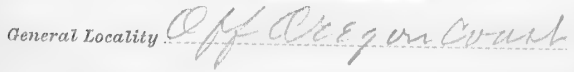

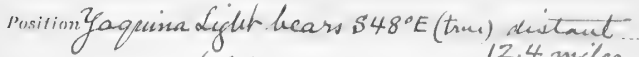

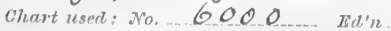

Drift: Dinertion

Disturue

Depth of Haul

Apparatus and Rig tused 
- A M. DOWN. UI'.

. 1 .

$6|4|$

643
Fass.

M.

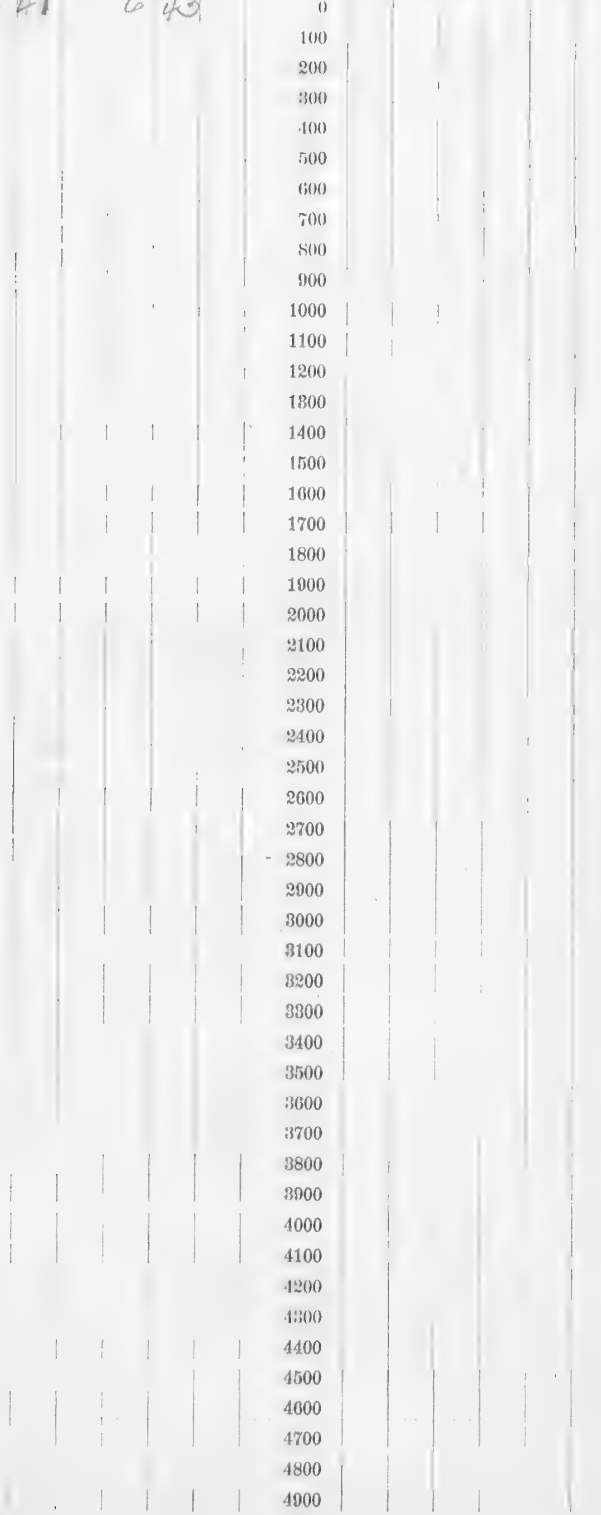




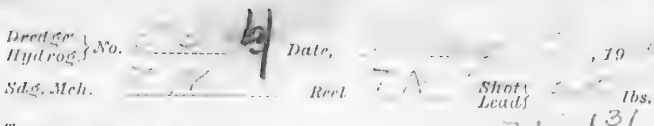

Turns

('in's'n Depth 131 Sals. Oup

Character of

Bottom

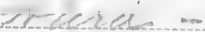

Thermom. $\left\{\begin{array}{l}\text { Alake } \\ \text { No. }\end{array}\right.$

rior'n $\left\{\begin{array}{l}\text { Instr. } \\ \text { Strm. }\end{array}\right.$

Bollom Readins

(Cor'd. Trmp).

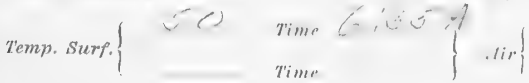

Density: Surf. fins.

Wind: Direction $5 \& \times E$ moree 2

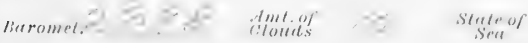

Weather ....... $C>7$

Direction and Force
of Gurrent.

Tils:

Aoneral lonatily

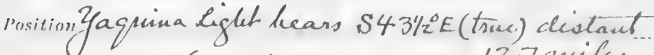
Whart user: No. 6000 $\pi l^{\prime}, n$

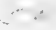

Irift: Mirertion

Distune?

Denth of Haul

Apparatus and Ris used

Remurls: 
9 M. DOW I Un. TIS.
(c) 5135

M. Jows. U1. M.

100

200

:3100

100

500

(6)0

700

: $:(1)$

900

1000

1100

1200

1300

1400

1500

1600

1700

1800

1900

2000

2100

2200

2300

2400

2500

2000

2700

2800

2900

3000

3100

3200

3300

3400

3500

3000

3700

3800

3900

1000

4100

4200

4300

4400

4500

4600

$17(x)$

4800

4000

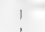

1

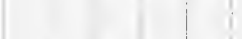

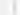

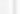


Mindiros Sits.uth. $7 / V$

atr.

\section{Turns}

Cor'n

Sils. ('u) מint $T$

charater of Bottom

\section{Amt, Bot'm}

$$
\text { Depth...7 | } \begin{aligned}
& 13 / \\
& \ldots \text { fms. }
\end{aligned}
$$

$\begin{aligned} & \text { Shot } \\ & \text { Lead }\}\end{aligned}-137^{\text {bs }}$

Whermom. $\left\{\begin{array}{l}\text { Hak" } \\ \text { Nin. }\end{array} \quad\right.$ Cor'n $\left\{\begin{array}{l}\text { Instr. } \\ \text { stem. }\end{array}\right.$

Bottom Reading Cor'd Temp.

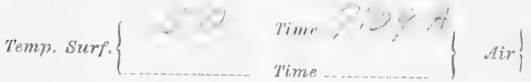

Dertsity: Surf.

fins.

Iriml: firction

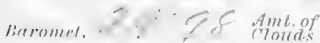

korer :

Weather of C $\geqslant x$

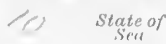

............

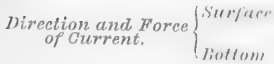

Tille

aeneral tocatity $C$

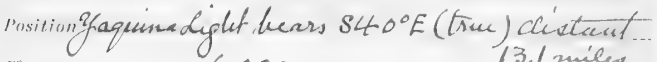

Chart used: No 6000

E'd't

Mrift: Direction

Distalure

Drepth of Hriul

Apnaratus and Rig used.

Remarks: 
is

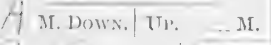

INA IREDGING CABLE.

$\int \mathrm{dit} \cdot \sqrt{\mathrm{x}^{2}}$

_.... M. Down.| UP. . MI.

$2(1)$

$: 3(1)$

(10)

$5(1)$

(i) (1)

ร)(1)

fi())

())

1000

1100

1:())

1300

1400

1500

1600

1700

1,800

$19(2)$

$\because 2()(0)$

2100

230()

2:360)

$\because 100$

$2=0$

2600

ערוt

39:

$\sin (9)$

$: 3(1)$

$\$ 100$

:2:(1)

: : $:(x)$

3100

(3)

:) (i)

:ละกแ

ing()

:3!(1)

(1)(1)()

1f(0)

(19)

(1) 1300

4:40

(15)10)

4(t)

(1)

(1800)

(4)(0) 




Wind: Direction ...5E

Direction and Force
of Current. $\left\{\begin{array}{l}\text { Surface } \\ \text { But um }\end{array}\right.$

Tile

General Lorertity

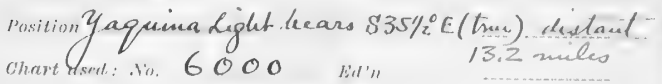
Drift: Direction Distance.

Depth of lazed.

Apparatus and Rig used

Time lowing

Dementia:

Derek. ofptere?

$\therefore$ Vieroriter

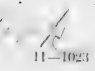


A. Mow. U1. MAS.

DREDGING CABLE.

$\because 25$

(1)

.... M. Down.I UP. _. M.

101)

200

(3)(1)

(10)

50()

(i)

700

800

900

1000

1100

1200

1300

1400

1500

1600

1700

1800

1800

2000

2100

2200

2300

2400

2500

2000

2700

2800

2900

3000

3100

3200

3300

3400

3500

$: 3600$

3700

$: 3800$

3900

1000

4100

4200

4:300

4400

.1500

4600

4700

4.800

4900 


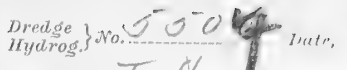
Solg. Mch. T

Turns Cor'n

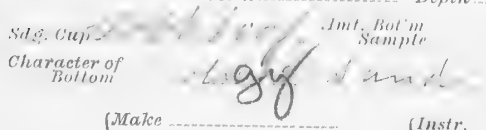

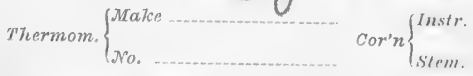

liottom linating

(for'd Timp).

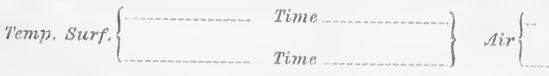

t)ensily: Sul.

firts.

Wind: Direclion

Force

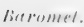

Amt of

\section{State of}

Howthrer

Direction and Force
of Current. $\left\{\begin{array}{l}\text { Surfiuep } \\ \text { Diollom, }\end{array}\right.$

ricles

General tooftity

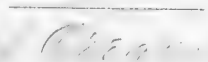

"ositionzaquina Light hears $\$ 311_{2}^{\circ}$ E (true) distant Chart used: No, 6000 E'/ ' '

Hrifl: Hirrelion

Jistante

Depth of Haul.

Apparatus and Ris used....

limmorls: 
SOUNDING WIRE.

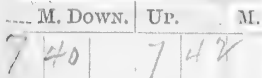

พMs.

DREDGING CABLE.

M. DOWN.| UP. M,

()

1(1)

2010

(301)

II)

into

(iti)

7(0)

$R(1)$ )

(10)

1000

110)

12(3)

1200

1.100

1500

14i(j)

1700

1.900

1900)

2000

2100

2900

aiso)

2100

$3+500$

:D(i)

$2 \pi(1)$

जe(0)

at)(a)

:alou

3100

:3) (1)

:2:30)

ह1(1)

Bin(1)

:ifit)

:3(1)

早i()()

:1)

f()

H1)(1)

$1: 3)$

lini)

111)( )

fin()

Ifil)

If()

ision

1900) 


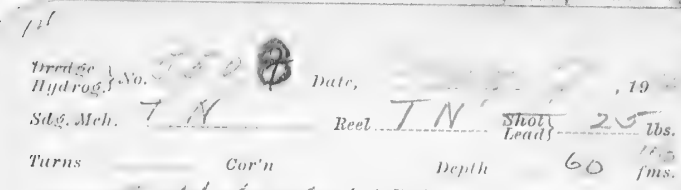
Character of Bollom
Thermom. $\left\{\begin{array}{l}\text { Wakt } \\ \text { No. }\end{array}\right.$
ogle w

Bottom Reading.

Cor'd Termp.

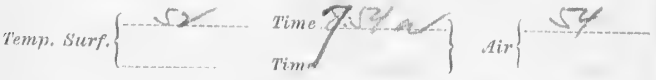
INosit!l: Surf:

fims.

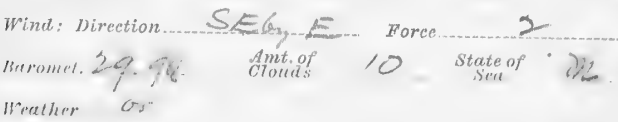

Direction and Force
of Ourrent.

Till"

Cenerat Lowrity

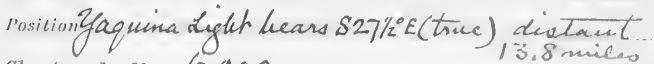
chart used: No... Ca a o , $k d$ 'n

1),rifl: Himetion

Distamen

Depth of Haut.

Apparatus and Rig used ....

Time Lowins

h.

$m$.

liemitrlis:



Wet isth Dy inll s.s. 0 5.8 .0 
SOUNDING WHIRE.

A M. DOw. Ui. A . I. $y-y \quad 2 \quad \cdots$
FMS.

1)REDGING CABLE

... M. I)

100

$2(0)$

:30)

400

500

(iil)

700

S(1)

9(0)

1000

1100

1:20()

1800

1.100

1504)

$16(1)(0)$

$1 \% 00$

$1 \mathrm{R}(10)$

1900

$2(501)$

$21(1)$

22000

$2: 000$

(291)

2500

291010

2800

ख्या10

:?)

:(4)0

$: 3100$

:ง

:300

:1400

:iis()0

:350)

ลงก0

2400

:日)

เ(0)(11)

(1101)

f'?(3)

(1:300)

4100

(1)

$460)$

$1 \%(0)$

1800

4900) 
15.

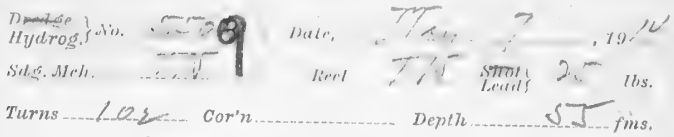

$$
\begin{aligned}
& \text { Sag. Cup ... Armings..... Amt. Bot'm } \\
& \text { Sample. }
\end{aligned}
$$

Character of
lint om!

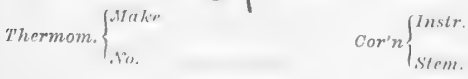

Bottom Reading

Cor'd Temp

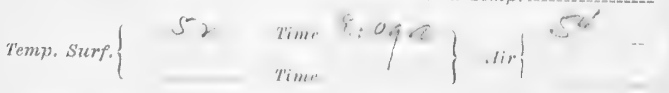

I)"usit!l: surf:

Wind: Direction S $5 \sigma_{2} E$ pore 2

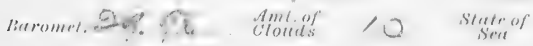

Weather

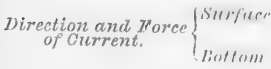

Tide

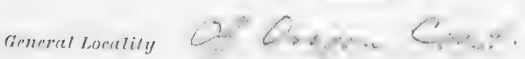

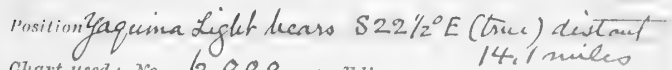
chart used: No 6000 Fin

Drift: Direction

Distance.

Depth of Haul

Apparatus and Rig used.

Timetourins

m.

lirmarks:

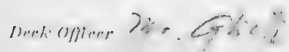

lietrintin.

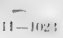


$11-1112$.

SOUNDING WIRE.

$\Rightarrow$ M. 1HWN.I U1. A M.

$8008 / 2$

FIIS.

I(t)

200

:300

100

500

(5)

700

s(1)

!)(6)

1000

1100

$10(4)$

13(3)

1.900

1500

16000

1700

1800

1900

2000

2100

$2: 00$

:2:30)

$\because 400$

500

2600

ขัตก

2900

2900

5000

$: 3100$

$\$ 200$

:1300

3100

3300

$\therefore 600$

:

: 2500

$\$ 300$

1000

1100

4200

4:300

1100

1.50)

1(tivo

1700

$1 \times 00$

f! $(100)$ 
16

$\left.\begin{array}{l}\text { Defied } \\ \text { IIydrog }\end{array}\right\}$ No.

Sdi. Meh.

Turns Le f Corn

Sag. Cup .

Character of

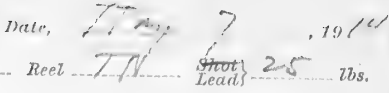
Depth $\sqrt{5}$ fms. t. Bot'm
Sample

gl:

Thermos, $\left\{\begin{array}{l}\text { Make } \\ \text { No. }\end{array}\right.$

Corn $\left\{\begin{array}{l}\text { Instr. } \\ \text { Stem. }\end{array}\right.$

Bottom Reading Cord Temp.

Temp. Surf. \{

Time Bizet

Time

Air

Density: Surf.

fins.

Wind: Direction... S AE E Force..

Q

suromel. $5 \%$. Cs

Amtiof

State of
sine

Weather cares

Direction and Force
of Current. $\left\{\begin{array}{l}\text { Surfer } \\ \text { bottom }\end{array}\right.$

Till:

General Locality

Oran Cont

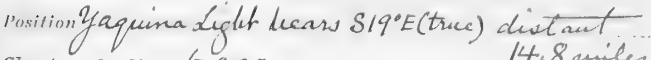
chart use it: No. Gad Han 14.8 miles

Drift: Direction Distance.

7)epth of Haul

Apparatus and Rig used

Fine torero:-

h. $m$.

Rimerka:

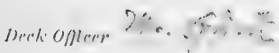

lierorder

(7) - - : 


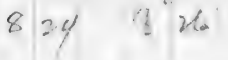

..... M. Down.l Ur. _....M.

(i)

()(i)

1)(1)0

1100

1:0)

1300

1.400

1500

$1600)$

$1 \% 00$

1800

1900

$20(0)$

2100

2200

$2: 300$

$\because 100$

2500

$\because 000$

2700

2800

2900

$: 3000$

3100

$\because \because(0)$

:3:300)

3100

$350(0)$

:a(i)o

$: 300$

$: 3800$

:日)

f(0)10

.1100

1.00

1300

4400

1500

4000

4700

$\sin (0)$

4900 


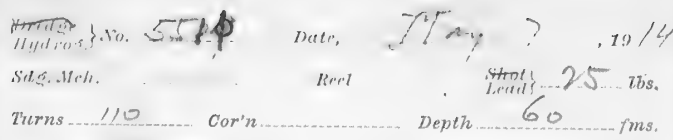

Sals. Ciup Gl......, Amt. Bot'm



Bottom Reading -...

Cor'd T'emp.

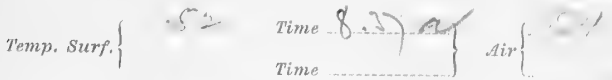

Density: Surf.

fins.

Wind: Direction..... $\leqslant-\frac{1}{2}$

Fioref

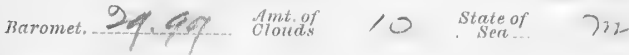

Ireather an. or

Direction and Worce
of Current. $\left\{\begin{array}{l}\text { Surfur" } \\ \text { Bottom }\end{array}\right.$

Tirle.

General Locality

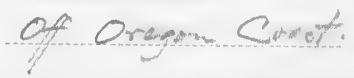

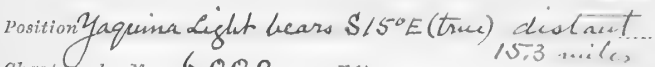
chart Ased: No, 6000 Ed'n

7)rift: Direction

Distance

Depth of Haut

Apparatus and Ris used.

limmirk:

werkonler ilir. $\Leftrightarrow \therefore i$

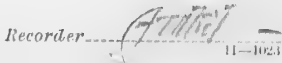


SUUTNI)IN(4 TVIRE.

तो M. Down. Uip. ?... M. $(1)+\ldots|q| \ldots \mid$

FMS.

DNEDGING CABLE

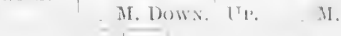

100

20()

$: 300$

.100

500

(it)o

;0)

$800)$

()() ()

$1(1)()$

1100)

1900

$1: 300$

1.100

1500

tition

1700

1:00

1900

$: 000$

2100

200

$2: 100$

$\because 100$

$\therefore \rightarrow(00)$

2600

3)

29:00)

2900

$: 000$

$\$ 100$

$: 3: 00$

3300

$3100)$

3500

$: 1600$

:300

38(0)

3901)

$.1000)$

4100

4200

4:300

$+100$

4.501)

4600

1700

4800

|19(4) 
18

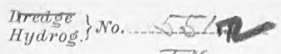

Date, .... 714.1914

Sd g. Meh.

Reel

Sweat
Lead

lbs.

Turns 27 Corn Depth fms.

Sag. Cup. Amt, Bot'm

Character of

Thermos. $\left\{\begin{array}{l}\text { Make } \\ \text { No. }\end{array}\right.$

ga 5

Bottom Reading

Corn $\left\{\begin{array}{l}\text { Instr. } \\ \text { Stem. }\end{array}\right.$

Temp. Surf. $\left\{\begin{array}{c}5 \% \\ \ldots\end{array}\right.$ Cord Temp.

Density: Surf. fins.

Wind: Direction Time 8.53 Time

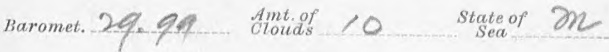
Weather op

Direction and Force $\left\{\begin{array}{l}\text { Surface } \\ \text { of Current. } \\ \text { Bottom. }\end{array}\right.$

Tide

General Locality ...... C24 Cone sente

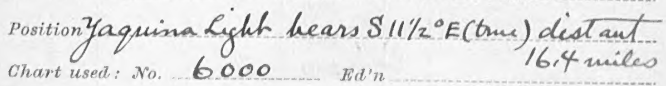

Drift: Direction Distance

Depth of Haul

Apparatus and Rig used.

Time towing h. $m$.

Remarks :

Deck officer Mho. Gites

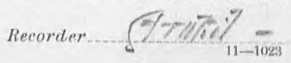


$11-1023$

SOUNDING WIRE.

\& M. Down. UP. Af. 853
FMS.

DAFEDGING CABLE.

M. Down. UP. M.

0

100

200

300

400

500

600

700

800

900

1000

1100

1200

1800

1400

1500

1600

1700

1800

1900

2000

2100

2200

2800

2400

2500

2600

2700

2800

2900

3000

8100

3200

3300

8400

8500

3600

8700

3800

3900

4000

4100

4200

4800

4400

4500

4600

4700

4800

4900
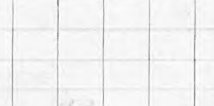

.


19

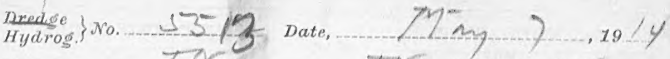

Sdg.Mch.

Reel $7 \sqrt{\text { Shot }} 25$ toad 2.

Turns 95 Corn

Depth 51 ins.

Sig. Cup Amt, Bot'm

Character of

Thermom. $\left\{\begin{array}{l}\text { Make } \\ \text { No. }\end{array}\right.$

Corn $\left\{\begin{array}{l}\text { Instr. } \\ \text { Stem. }\end{array}\right.$

Bottom Reading

Cor'd Temp.

Temp. Surf. \{

Time $q \circ\}$

Time

Density: Surf.

fins.

Wind: Direction

Force

$1-2$

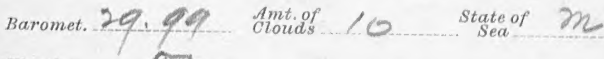

Weather

Direction and Force
of Current. $\left\{\begin{array}{l}\text { Surface } \\ \text { Bottom }\end{array}\right.$

Tide

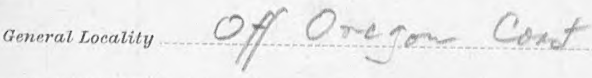
Position Yaquina Light hears S10\% ${ }^{\circ}$ E( the) distant
17.2 miles chart used: No. 6000_... ERin

Drift: Direction

Distance

Depth of Haul

Apparatus and Rig used

Time towing 7. $m$.

Remarks:

Deck offer Mr. Pick

Recorder

11-1023 
